\title{
Oncogenic TYK2 P760L kinase is effectively targeted by combinatorial TYK2, mTOR and CDK4/6 kinase blockade
}

\author{
Katharina Woess, ${ }^{1}$ Sabine Macho-Maschler, ${ }^{2}$ Dorette S. van Ingen Schenau, ${ }^{3}$ Miriam Butler, ${ }^{3}$ \\ Caroline Lassnig,, ${ }^{1,4}$ Daniel Valcanover, ${ }^{1}$ Andrea Poelzl, ${ }^{4}$ Katrin Meissl, ${ }^{1}$ Barbara Maurer, ${ }^{5}$ Tania \\ Brandstoetter, ${ }^{5}$ Claus Vogl, ${ }^{1}$ Anna Koren, ${ }^{6}$ Stefan Kubicek, ${ }^{6}$ Anna Orlova,, Richard Moriggl, \\ Birgit Strobl, ${ }^{1}$ Veronika Sexl, ${ }^{5}$ Frank N. van Leeuwen, ${ }^{3}$ Roland P. Kuiper ${ }^{3,7}$ and Mathias \\ Mueller ${ }^{1,4}$
}

${ }^{1}$ Institute of Animal Breeding and Genetics, University of Veterinary Medicine Vienna, Vienna, Austria; ${ }^{2}$ Unit of Physiology, Pathophysiology and Experimental Endocrinology, University of Veterinary Medicine Vienna, Vienna, Austria; ${ }^{3}$ Princess Máxima Center for Pediatric Oncology, Utrecht, the Netherlands; ${ }^{4}$ University Center Biomodels Austria, University of Veterinary Medicine Vienna, Vienna, Austria; ${ }^{5}$ Institute of Pharmacology and Toxicology, University of Veterinary Medicine Vienna, Vienna, Austria; ${ }^{6} \mathrm{CeMM}$ Research Center for Molecular Medicine of the Austrian Academy of Sciences, Vienna, Austria and ${ }^{7}$ Department of Genetics,

University Medical Center Utrecht, Utrecht, the Netherlands

\author{
Correspondence: M. Mueller \\ mathias.mueller@vetmeduni.ac.at \\ Received: \\ Accepted: \\ September 7, 2021. \\ January 5, 2022 \\ Early view: \\ January 13, 2022.
}

https://doi.org/10.3324/haematol.2021.279848

(อ2023 Ferrata Storti Foundation

Published under a CC BY-NC license (c) (1) ()

\begin{abstract}
Tyrosine kinase 2 (TYK2) is a member of the Janus kinase/signal transducer and activator of transcription pathway, which is central in cytokine signaling. Previously, germline TYK2 mutations have been described in two patients developing de novo T-cell acute lymphoblastic leukemias (T-ALL) or precursor B-ALL. The mutations (P760L and G761V) are located within the regulatory pseudokinase domain and lead to constitutive activation of TYK2. We demonstrate the transformation capacity of TYK2 P760L in hematopoietic cell systems including primary bone marrow cells. In vivo engraftment of TYK2 P760L-expressing cell lines led to development of leukemia. A kinase inhibitor screen uncovered that oncogenic TYK2 acts synergistically with the PI3K/AKT/mTOR and CDK4/6 pathways. Accordingly, the TYK2-specific inhibitor deucravacitinib (BMS986165) reduces cell viability of TYK2 P760L-transformed cell models and ex vivo cultured TYK2 P760L-mutated patient-derived xenograft cells most efficiently when combined with mTOR or CDK4/6 inhibitors. Our study thereby pioneers novel treatment options for patients suffering from TYK2-driven acute leukemia.
\end{abstract}

\section{Introduction}

The Janus kinase (JAK)/signal transducer and activator of transcription (STAT) pathway links extracellular cytokines with transcriptional regulation and reprogramming. As a core cancer pathway ${ }^{1}$ it connects to other cancer-driving signaling cascades such as the PI3K/AKT/mTOR or cell cycle progression pathways. ${ }^{2-4}$ Alterations in JAK/STAT signaling are associated with cancer and immune system disorders. ${ }^{5}$ Activating JAK $1 / 2 / 3$ mutations are frequent in various hematologic malignancies, whereas the role of the JAK family member tyrosine kinase 2 (TYK2) has only recently emerged. ${ }^{6-8}$ More evident are TYK2 mutations causative for immune system disorders and inflammatory diseases. ${ }^{9}$

TYK2 is associated with the cytokine family receptors for type I interferon (including interferon [IFN]- $\alpha$ and $-\beta$ ), interleukin (IL)-12 (including IL-23) and IL-10 (including type III IFN, IL-22 and IL-26). Under physiological conditions TYK2 activity may provoke tyrosine phosphorylation of all
STAT (STAT1-6). ${ }^{10}$ The sparse reports on aberrant TYK2 activity in cancers include TYK2 locus mutations, fusion proteins and crosstalk to oncogenic pathways. ${ }^{8}$ The first somatic activating TYK2 mutations have been described in T-cell acute lymphoblastic leukemia (T-ALL) cell lines." TYK2 fusion proteins have been found in various hematologic malignancies ${ }^{12-14}$ and TYK2 cross-talks to the nucleophosmin-anaplastic lymphoma kinase pathway in anaplastic large cell lymphoma. ${ }^{15}$

Next-generation sequencing is revealing an increasing number of germline gene alterations predisposing to leukemia. ${ }^{16}$ The germline TYK2 mutations (P760L, G761V) were identified in pediatric patients who developed multiple de novo ALL: the TYK2 P760L carrying patient showed two precursor B-ALLs and the TYK2 G761V patient two T-ALL. Both mutations affect the pseudokinase domain (JAK homology [JH]2 domain) and cause constitutive activation of TYK2 and of downstream STAT1/3/5. ${ }^{17}$

The first JAK inhibitors developed acted by competing ATP binding at the tyrosine kinase (JH1) domain. The high homology of the kinase domain within the JAK family or 
other tyrosine kinases limits specificity of such inhibitors. ${ }^{18}$ The highly specific TYK2 inhibitor deucravacitinib acts differently and stabilizes the negative regulatory pseudokinase $\mathrm{JH} 2$ domain. ${ }^{19-21}$ The efficacy of deucravacitinib is currently evaluated in several advanced clinical trials for the treatment of autoimmune/inflammatory diseases. ${ }^{20,21}$ Combining inhibitors is beneficial, as combinatorial treatments allow for reduced drug concentrations to avoid side effects and may overcome potential resistances. ${ }^{22}$ Notably, JAK1/2/3 inhibitors in single or combined drug treatments are already applied for hematological malignancies. ${ }^{23}$

We here characterized TYK2-activating germline mutations (P760L, G761V) that have been described in childhood leukemia patients with respect to their transformation potential in vitro and in vivo. The TYK2 P760L mutation efficiently transforms hematopoietic cells and provokes cancer upon transplantation in mice. Viability of TYK2transformed cells is efficiently reduced by the highly specific TYK2 inhibitor deucravacitinib. A comprehensive inhibitor-based screen identified pathways co-operating with oncogenic TYK2. We show synergistic action of deucravacitinib with inhibitors of the top hit pathways PI3K/AKT/mTOR or CDK4/6 in TYK2 P760L-transformed cell lines and in the TYK2 P760L-mutated patient-derived xenograft (PDX) cells. This reveals novel treatment options for acute leukemia in patients harboring gain-of-function (GOF) TYK2.

\section{Methods}

Details on plasmids, generation and cultivation of cell lines and primary cells, cell viability assays, flow cytometry, immunoblotting and histochemistry are described in the Online Supplementary Appendix.

\section{Ethics statement}

Mice were housed under specific pathogen-free conditions according to FELASA guidelines. Animal experiments were approved by the Institutional Ethics and Animal Welfare Committee of the University of Veterinary Medicine Vienna, the Austrian authority according to $\S \S$ 26ff. of Animal Experiments Act, Tierversuchsgesetz 2012: TVG 2012 (BMBWF-68.205/0112-WF/V/3b/2016, BMBWF$68.205 / 0174-\mathrm{V} / 3 \mathrm{~b} / 2018$ ) and the Animal Experimental Committee of the Radboud University Medical Center (AVD1030020209324). PDX cells were generated by the Dutch Childhood Oncology Group. Informed consent for the use of spare specimens for research was obtained from study individuals, parents or legal guardians.

\section{Drug screen}

CellTiter-Glo Luminescent Cell Viability Assays (Promega, Madison, WI, US) in 384-well plates were performed in an automated high-throughput approach (CeMM Molecular Discovery Platform - Chemical Screening). Six hundred and eighty drugs of kinase inhibitor libraries (L1600, Targetmol, Boston, MA, US and 10505, Cayman Chemicals, Ann Arbor, MI, US) were screened at $10 \mu \mathrm{M}$ in duplicates on $2 \times 10^{3} \mathrm{Ba} / \mathrm{F} 3$ TYK2 P760L 1 cells/well for 3 days. Hits were selected having $<30$ percentage of control (POC) viability by setting the positive control to $0 \%$ (Bortezomib, $10 \mu \mathrm{M})$ and the negative control ( $0.1 \%$ dimethyl sulfoxide [DMSO]) to $100 \%$. Two hundred and forty-six drugs from the first round were screened in triplicates with four different 10-fold dilutions on Ba/F3 TYK2 P760L 1 and parental Ba/F3 cells (cultivated with $1 \mathrm{ng} / \mathrm{mL} / \mathrm{L}-3$ ) for 3 days. For hit calling the mean difference of the area under the curve between Ba/F3 TYK2 P760L 1 and parental Ba/F3 cell POC curves was calculated.

\section{In vivo experiments}

Male and female age-matched (8-22 weeks) NOD.Cg-

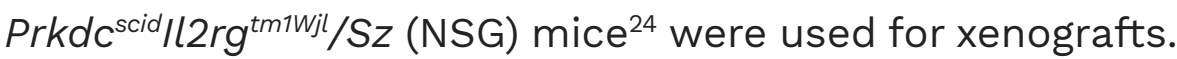
Bone marrow (BM)-derived patient cells were washed with phosphate-buffered saline (PBS) and digested with DNAse I (130 $\mu \mathrm{g} / \mathrm{mL}$, Roche, Basel, Switzerland) for 10 minutes (min) at room temperature. $5 \times 10^{5}$ cells were injected intrafemorally (Radboud University Medical Center, Nijmegen, the Netherlands). PDX cells in the blood were assessed bi-weekly and upon detection of human cells weekly. Mice were sacrificed once human cells reached $>50 \%$ or at the humane endpoint (defined by scoring appearance, behavior, posture and mobility).

$\mathrm{Ba} / \mathrm{F3}$ and 32D cell lines were washed with PBS. $1 \times 10^{6}$ cells were injected intravenously, $5 \times 10^{6}$ cells subcutaneously. For survival studies mice were sacrificed at the humane endpoint (see above and for subcutaneous injection a tumor volume of $1,500 \mathrm{~mm}^{3}$ ). Tumor volume (=length*width ${ }^{2} / 2$ ) was measured with a caliper.

Organs were weighed and histologically examined. Blood parameters were measured by VetABC (scil, Viernheim, Germany). Blood was incubated with an ammoniumchloride-potassium lysis buffer for $5 \mathrm{~min}$. Solid tissues were mashed through a $100 \mu \mathrm{m}$ nylon cell strainer and red blood cell lysis was performed for spleen and liver. All isolated cells were analyzed by flow cytometry or cryopreserved.

\section{Statistical analysis}

One-way or two-way ANOVA with Tukey post hoc test and unpaired two-sided $t$-tests were performed on log or arcsine square root transformed data using GraphPad Prism version 7.0 for Mac (GraphPad Software, San Diego, CA, US). Mean \pm standard deviation and statistical significance are shown $(* P<0.05, * * P<0.01, * * * P<0.001, * * * * P<0.0001)$. 


\section{Results}

TYK2 P760L induces colony formation of primary bone marrow cells

We transduced primary murine BM cells with a retrovirus encoding human TYK2 constructs (Figure 1A) to study the effect of TYK2 P670L on colony formation. In order to test for TYK2-intrinsic kinase activity we introduced M978F as a kinase-inactivation mutation ${ }^{11}$ in the expression constructs. Co-expression of green fluorescent protein (GFP) allowed for the assessment of the transduction efficiency. On average $15 \%$ of cells were retrovirally transduced (Online Supplementary Figure S1) and an equal number of $\mathrm{GFP}^{+}$cells was seeded into growth factor-free methylcellulose. TYK2 P760L-transduced BM cells formed colonies at largest size (Figure 1B) and highest number compared to cells expressing wild-type (WT) TYK2, TYK2 P760L, M978F or empty GFP vector (EV) (Figure 1C). TYK2 P760L- expressing BM cells were capable of replating for two rounds indicative for an enhanced self-renewal capacity (Figure 1C). The majority of the GFP ${ }^{+}$TYK2 P760L-transduced BM cells showed characteristics of hematopoietic stem/progenitor cells (Lin- Sca- $\left.1^{+} \mathrm{C}-\mathrm{Kit}^{+}\right)^{25}$ (Figure 1D).

These data led us to conclude that TYK2 P760L enables colony formation of primary BM cells in growth factor-free conditions.

\section{TYK2 P760L allows factor independence of} hematopoietic cell lines and leukemogenesis in vivo In order to further assess the ability of TYK2 mutants to provide growth factor independence, we used various hematopoietic cell lines including the stem cell factor (SCF)-dependent hematopoietic progenitor cell line HPC7, the IL-3-dependent pro-B cell line $\mathrm{Ba} / \mathrm{F} 3$ and the BMderived progenitor cell line 32D.

In this experimental setting we employed the TYK2 P760L
A

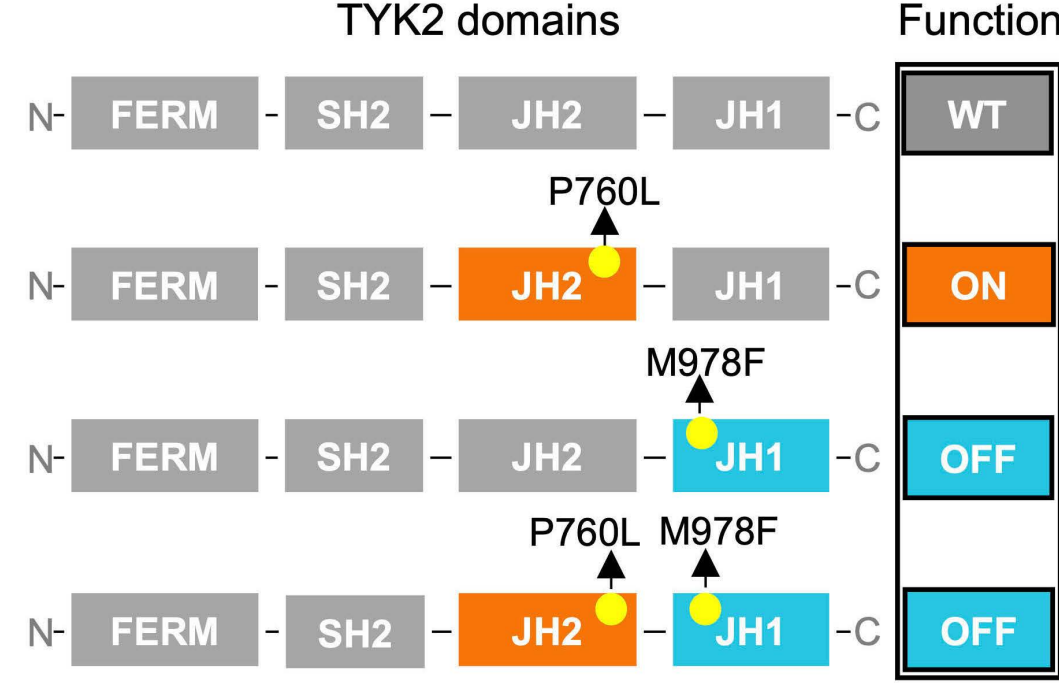

B WT TYK2 TYK2 ${ }^{\text {P760L }} \quad$ TYK2 ${ }^{\text {P760L, M978F }}$ EV

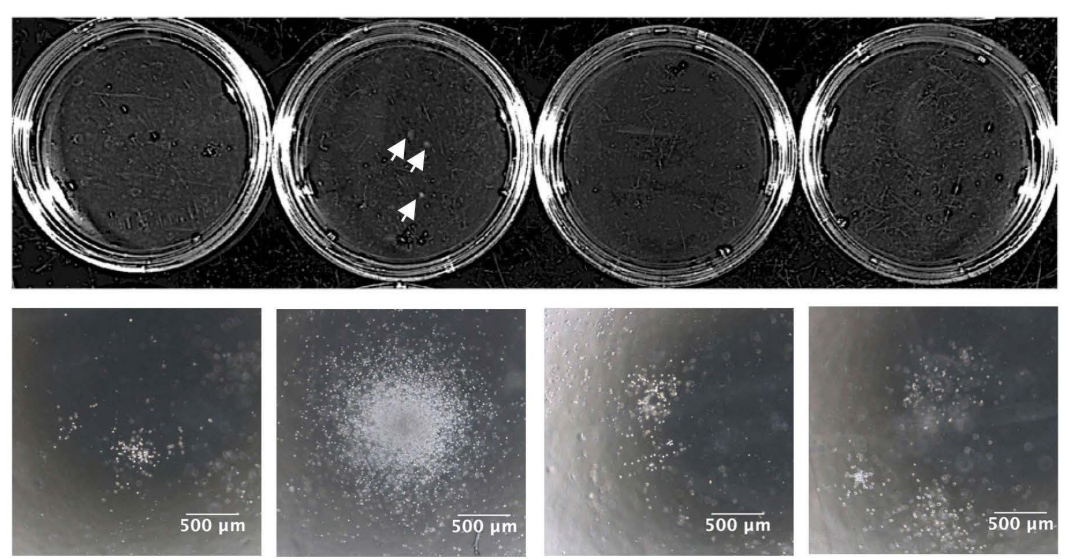

C

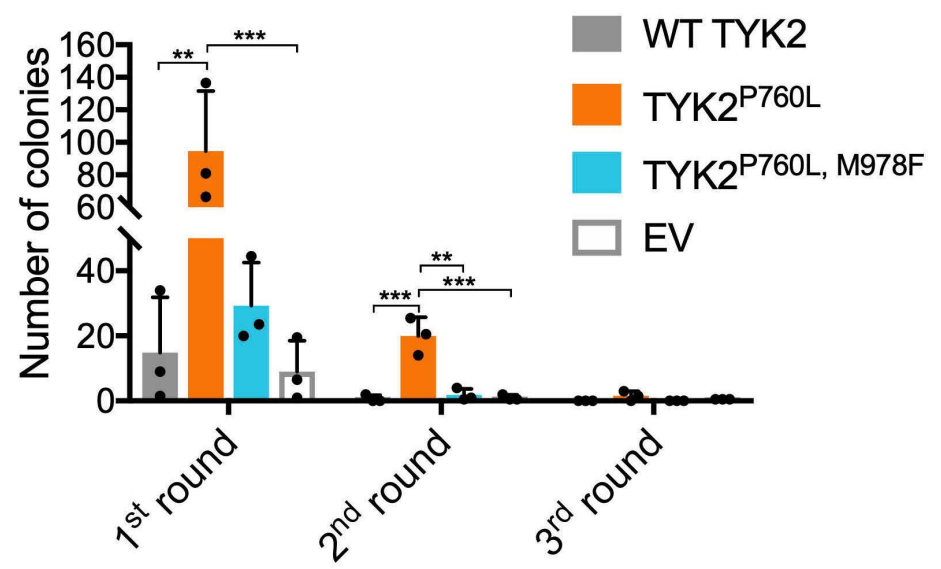

D TYK2 $2^{\text {P760L }}$

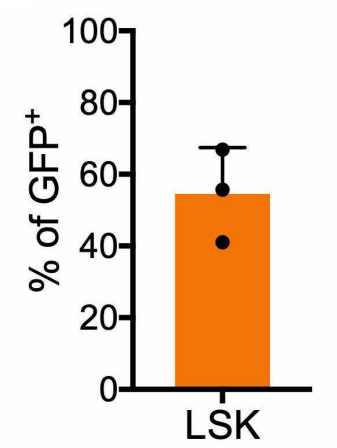

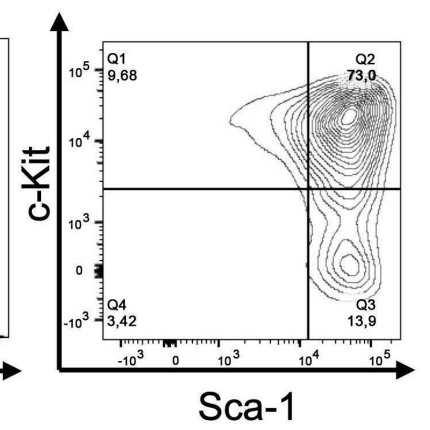

Figure 1. TYK2 P760L-transduced primary bone marrow cells and factor-free colony formation. (A) Schematic illustration of combinations of kinase-activating germline and kinase-inactivating mutations: TYK2 consists of a four-point-one, ezrin, radixin, moesin (FERM), an atypical Src-homology 2 (SH2), a JAK homology (JH)2 and a JH1 domain. In wild-type (WT) TYK2 the JH2 pseudokinase domain inhibits the $\mathrm{JH} 1$ kinase domain at basal state; the $\mathrm{JH} 1$ domain is activated upon cytokine receptor engagement (WT, grey). P760L alters the structure of a conserved motif in the JH2 domain which is predicted to attenuate the kinase inhibitory $\mathrm{JH} 2-\mathrm{JH} 1$ interaction (ON, orange). M978F inactivates the $\mathrm{JH} 1$ domain (= OFF, blue). Combination of M978F with P760L abolishes the TYK2 kinase activity. (B) Representative colony pictures of transduced bone marrow (BM) cells, taken with 4-fold magnification objective for single colonies. Scale bars show $500 \mu \mathrm{m}$. White arrows indicate visible colonies. (C) Replating experiment of transduced BM cells in factor-free methylcellulose ( $n=3$, duplicates, two-way ANOVA with log transformed data). (D) Percentage of $\mathrm{Lin}^{-}, \mathrm{Sca}-1^{+}, \mathrm{C}_{-} \mathrm{Kit}^{+}$(LSK) cells among GFP ${ }^{+}$TYK2 P760L-transduced BM cells after the first round in methylcellulose $(n=3)$. Cells were gated on single cells, living, GFP+, Lin', c-Kit ${ }^{+}$and Sca- $1^{+}$. Representative plots are shown. $\star * P<0.01, * * * P<0.001$. 
and the TYK2 G761V mutant, another activating germline TYK2 mutant identified in pediatric ALL. In order to study the activation status of the TYK2-STAT axis, retrovirally transduced and GFP ${ }^{+}$-sorted HPC-7 cells (Figure 2A) were starved for 6 hours (h) as SCF may activate components of the JAK-STAT signaling pathway. ${ }^{26}$ As previously shown ${ }^{17}$ TYK2 P760L and TYK2 G761V enhanced TYK2 phosphorylation and increased phospho-STAT1 and -STAT3 levels. The effects on phospho-STAT5A/B were less consistent. The ectopic overexpression of WT TYK2 also induced activation of STAT1 and STAT3. Introduction of the TYK2 M978F mutation in the expression cassettes showed that the phosphorylation events were a direct consequence of TYK2 kinase activity (Figure 2B). Both TYK2-activating mutants induced the outgrowth of colonies of the immortalized progenitor cell line HPC-7 supplemented with a reduced SCF concentration. Colony numbers raised upon replating to a greater extent in TYK2 P760L cells (Figure 2C; Online Supplementary Figure S2A). This effect did not translate into a growth advantage in suspension culture upon titrated reduction of SCF (Online Supplementary Figure $S 2 B)$.

Next, we electroporated the TYK2 expression cassettes into $\mathrm{Ba} / \mathrm{F} 3$ and $32 \mathrm{D}$ cells (Figure $3 \mathrm{~A}$ ). In both cell lines
TYK2 P760L expression enabled complete IL-3-independent growth while TYK2 G761V was only capable to transform 32D cells (Figure 3B; Online Supplementary Figure S3A). As in HPC-7 cells the TYK2 P760L-transformed cells showed constitutively activated TYK2-STAT1/3 (Online Supplementary Figure $S 3 B$ ). Taken together, both TYK2 germline mutations enhance STAT signaling in a TYK2 kinase-dependent manner and TYK2 P760L has a stronger transforming potential than TYK2 G761V in hematopoietic cell lines. The reasons for this remain to be elucidated. Notably, the activation state level mediated by TYK2 G761V seems to be lower than in TYK2 P760L-expressing cells as indicated by constitutive TYK2 or downstream STAT1 phosphorylation (Figure 2B and Waanders et $a l .{ }^{17}$ ).

In order to assess the in vivo oncogenic potential, we injected $\mathrm{Ba} / \mathrm{F} 3$ or 32D TYK2 P760L cells into NSG mice (Figure $3 \mathrm{~A}$ ) and used parental cells as controls. Upon systemic transplantation both cell lines harboring mutated TYK2 induced splenomegaly (Figure 3C; Online Supplementary Figure $\mathrm{S} 3 \mathrm{H}$ ) and led to high white blood cell counts (Figure 3D). Ba/F3 TYK2 P760L cells additionally caused enlarged livers (Figure 3E; Online Supplementary Figure $\mathrm{S} 3 \mathrm{H}$ ), thrombocytopenia (Figure 3F) and anemia (Figure 3G). As a consequence, the average survival of mice that had been
A

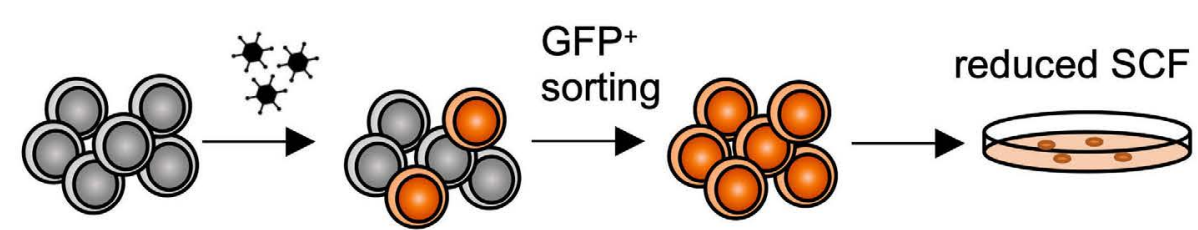

HPC-7

C

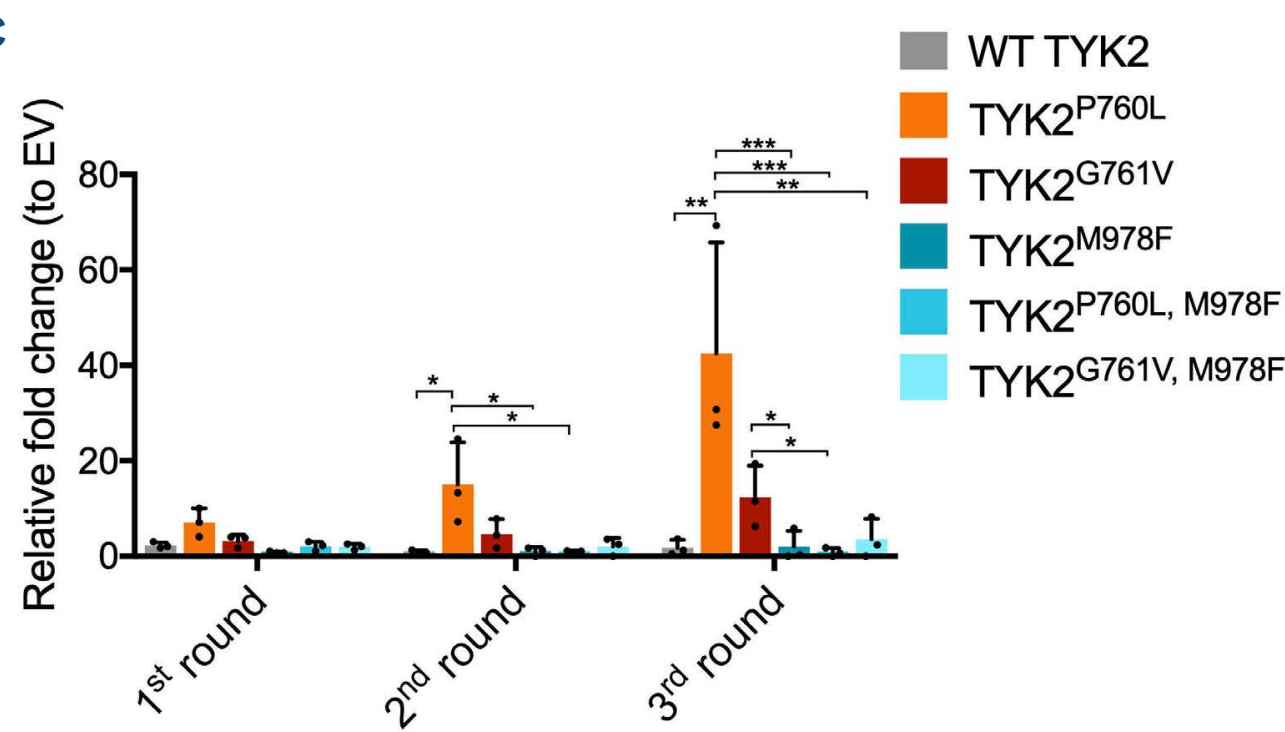

B

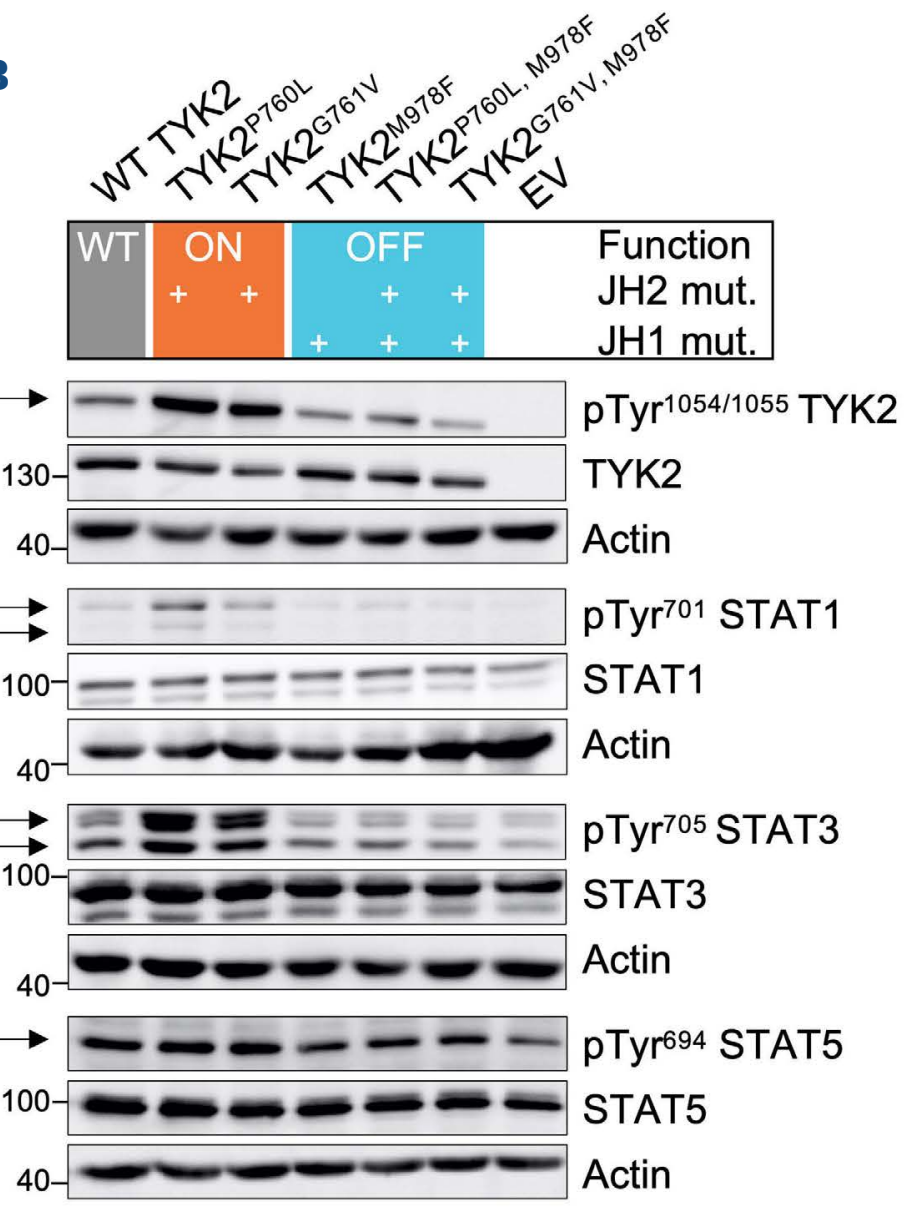

Figure 2. In vitro transformation capacity of TYK2 mutants. (A) Schematic outline of the HPC-7 cell experiment: cells were retrovirally transduced with the different TYK2 constructs (Figure 1A). HPC-7 cells were GFP ${ }^{+}$-sorted and seeded into

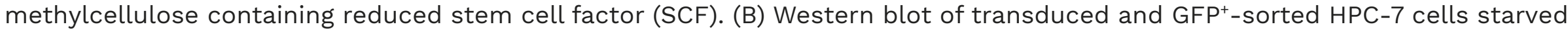
for SCF for 6 hours (h) subjected to (phospho-) TYK2 (human) and STAT1/3/5 (murine) analysis. Wild-type (WT) TYK2 activity can be regulated (grey), JH2 mutations activate TYK2 signaling (ON, orange), JH1 mutation inhibits TYK2 signaling (OFF, blue). Actin was used as loading control. Numbers indicate molecular weight markers in kDa. (C) Replating experiment of transduced and GFP ${ }^{+}$-sorted HPC-7 cells expressing WT TYK2 (grey), TYK2 P760L (orange), TYK2 G761V (red) or kinase inactive TYK2 (blue) in methylcellulose with reduced SCF ( $n=3$, two-way ANOVA with log transformed data, log colony number of empty vector (EV) transduced cells was subtracted from log number of TYK2-expressing colonies). ${ }^{\star} P<0.05, * \star P<0.01, * * \star P<0.001$. 

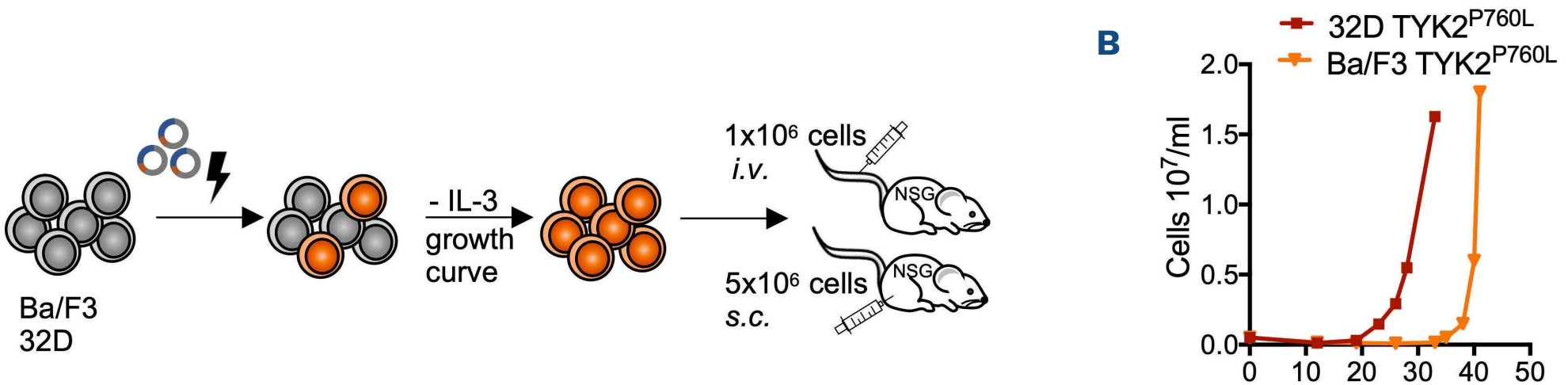

Days after IL-3 withdrawal

C

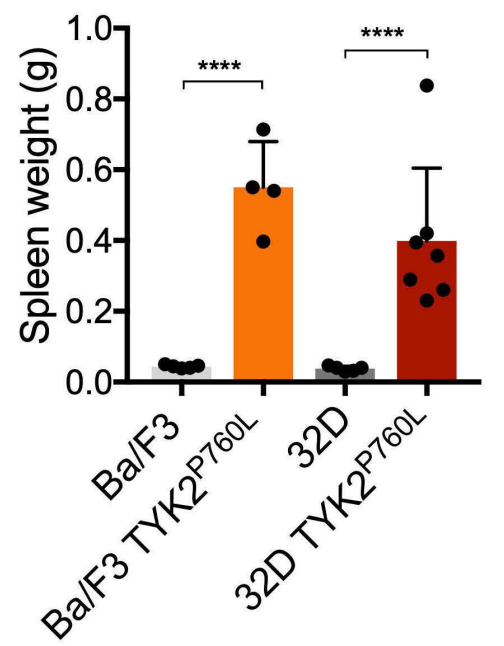

$\mathbf{F}$

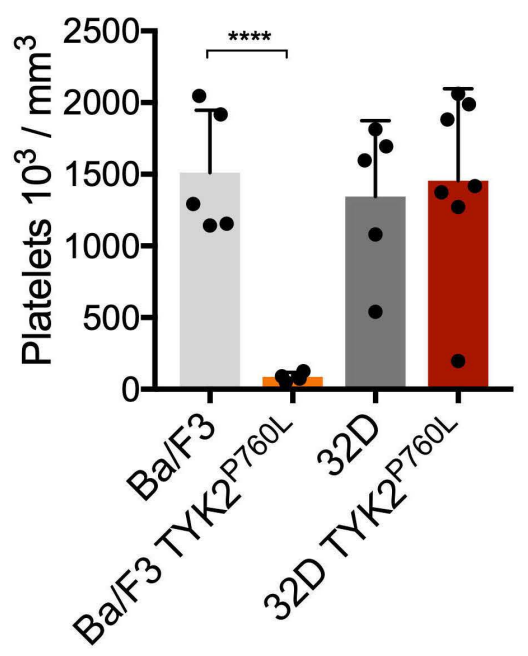

I

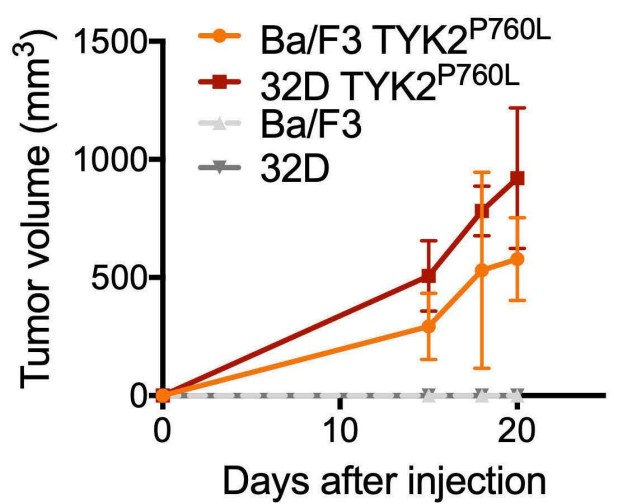

G

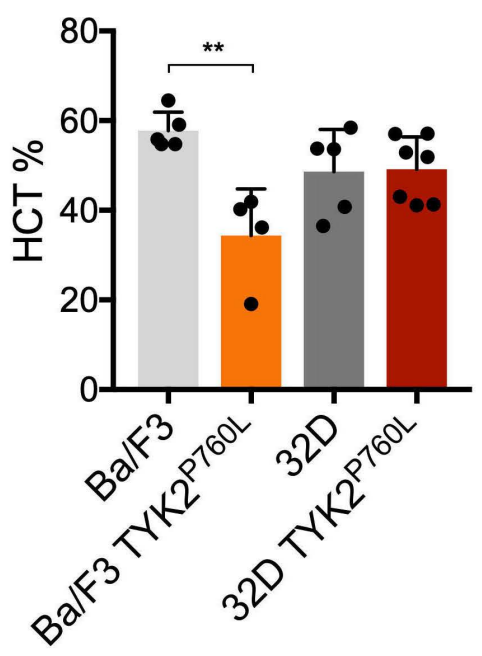

D $\stackrel{m}{E} \quad$ E

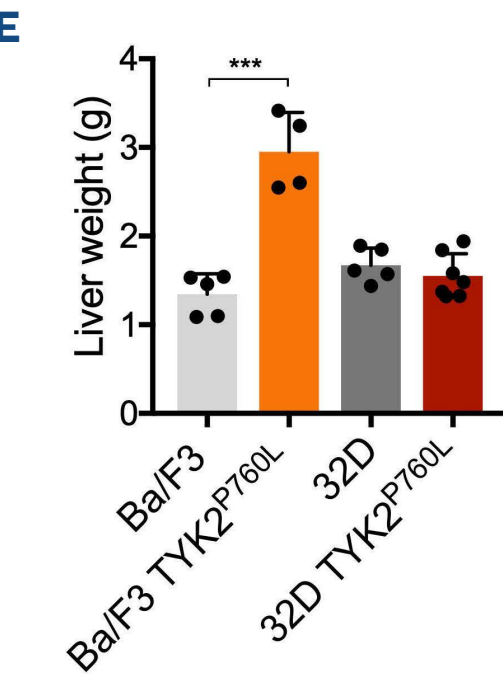

H

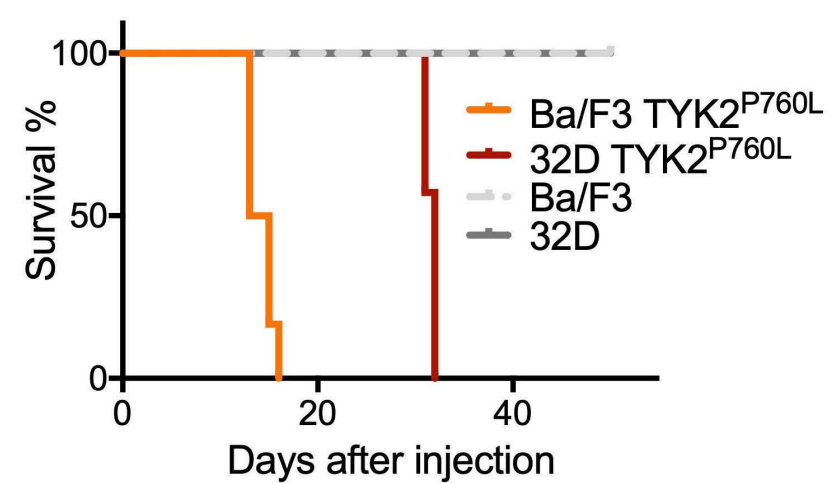

Figure 3. In vitro and in vivo transformation capacity of TYK2 mutants. (A) Schematic outline of the $\mathrm{Ba} / \mathrm{F} 3$ and 32D cell experiment: cells were electroporated with the different TYK2 constructs (Figure 1A), IL-3 was withdrawn, and outgrowth was monitored. Transformed and parental cells were injected intravenously (i.v.) or subcutaneously (s.c.)

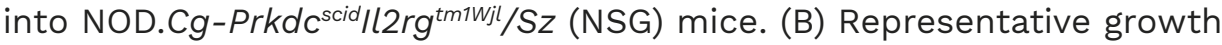
curve of $\mathrm{Ba} / \mathrm{F} 3$ (orange) and $32 \mathrm{D}$ cells (red) gaining IL-3 independence by expression of TYK2 P760L $(n=3)$. ( $C$ to $G)$ Spleen weight with representative spleen pictures, white blood cell count, liver weight, platelet count and hematocrit (HCT) of i.v. injected mice (Ba/F3 and 32D cells: $\mathrm{n}=5, \mathrm{Ba} / \mathrm{F} 3$ TYK2 P760L cells: $\mathrm{n}=4$ (as humane endpoint was set for 2 mice), 32D TYK2 P760L cells: $\mathrm{n}=7$, unpaired two-tailed $t$-test with log and arcsine square root [HCT] transformed data, from 2 experiments). (H) Kaplan Meier plot of i.v. injected mice (Ba/F3 and 32D cells [grey]: $\mathrm{n}=5, \mathrm{Ba} / \mathrm{F} 3$ TYK2 P760L cells: $\mathrm{n}=6,32 \mathrm{D}$ TYK2 P760L cells: $\mathrm{n}=7$, log-rank test: $P$-value $=0.0002$ between $\mathrm{Ba} / \mathrm{F} 3$ TYK2 P760L cells and 32D TYK2 P760L cells, from 2 experiments). (I) Tumor growth of s.c. injected cells into right and left flank (Ba/F3 TYK2 P760L cells: $\mathrm{n}=5$ [individual tumors, one site without tumor formation], 32D TYK2 P760L cells: $n=6$ [individual tumors]) and individual tumor weight (Ba/F3 TYK2 P760L cells: $\mathrm{n}=8$ [2 sites without tumor formation], 32D TYK2 P760L cells: $n=10$, from 2 experiments). ${ }^{*} P<0.01, * \star * P<0.001, * * * \star P<0.0001$. 
A

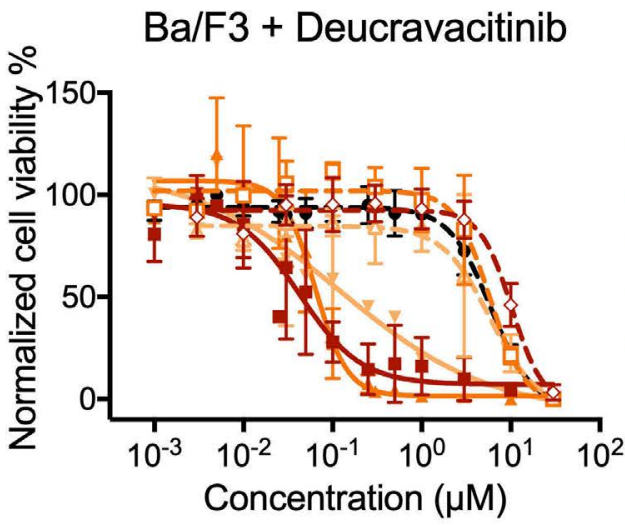

- Parental + IL-3

$-\mathrm{TYK}^{\mathrm{P} 760 \mathrm{~L}} 1$

- TYK2 ${ }^{\text {P760L }} 2$

$\div$ TYK2 ${ }^{\text {P760L }} 3$

$\curvearrowright$ TYK2 $2^{\mathrm{P} 760 \mathrm{~L}} 1+\mathrm{IL}-3$

$-\square-$ TYK2 $2^{\mathrm{P} 760 \mathrm{~L}} 2+\mathrm{IL}-3$

$\triangle$ TYK2 $^{\mathrm{P} 760 \mathrm{~L}} 3+\mathrm{IL}-3$

B

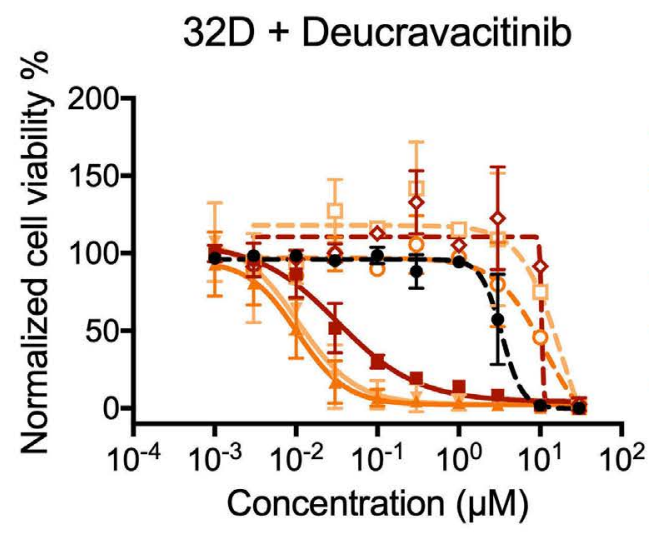

- Parental + IL-3

- TYK2 ${ }^{\mathrm{P} 760 \mathrm{~L}} 1$

$=$ TYK2 $2^{\text {P760L }} 2$

$\div$ TYK2 ${ }^{\text {P760L }} 3$

$\approx$ TYK2 ${ }^{\text {P760L }} 1+$ IL-3

$-\square-$ TYK2 $2^{P 760 L} 2+$ IL-3

$\triangle$ TYK2 $2^{\text {P760L }} 3+$ IL-3
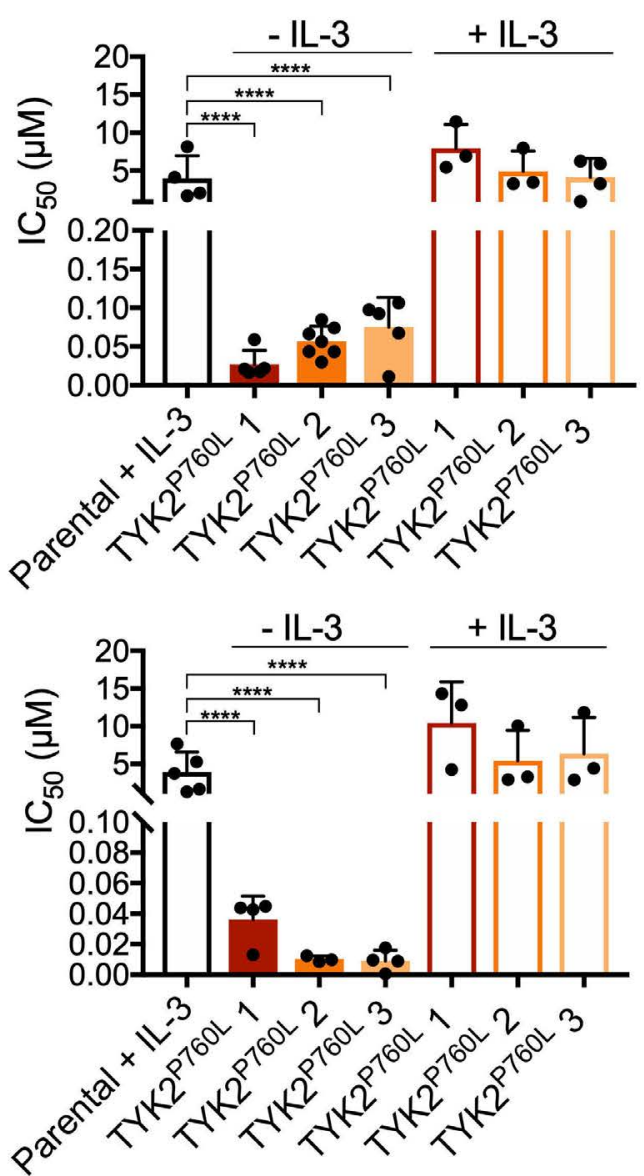

C
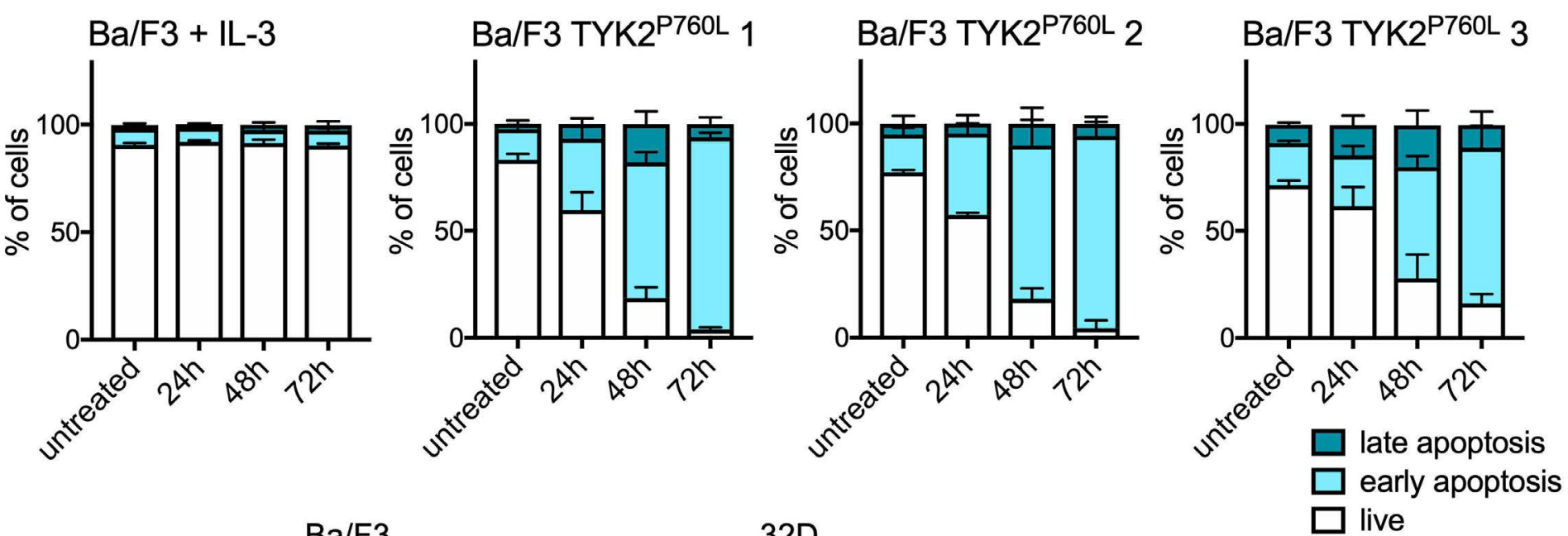

D
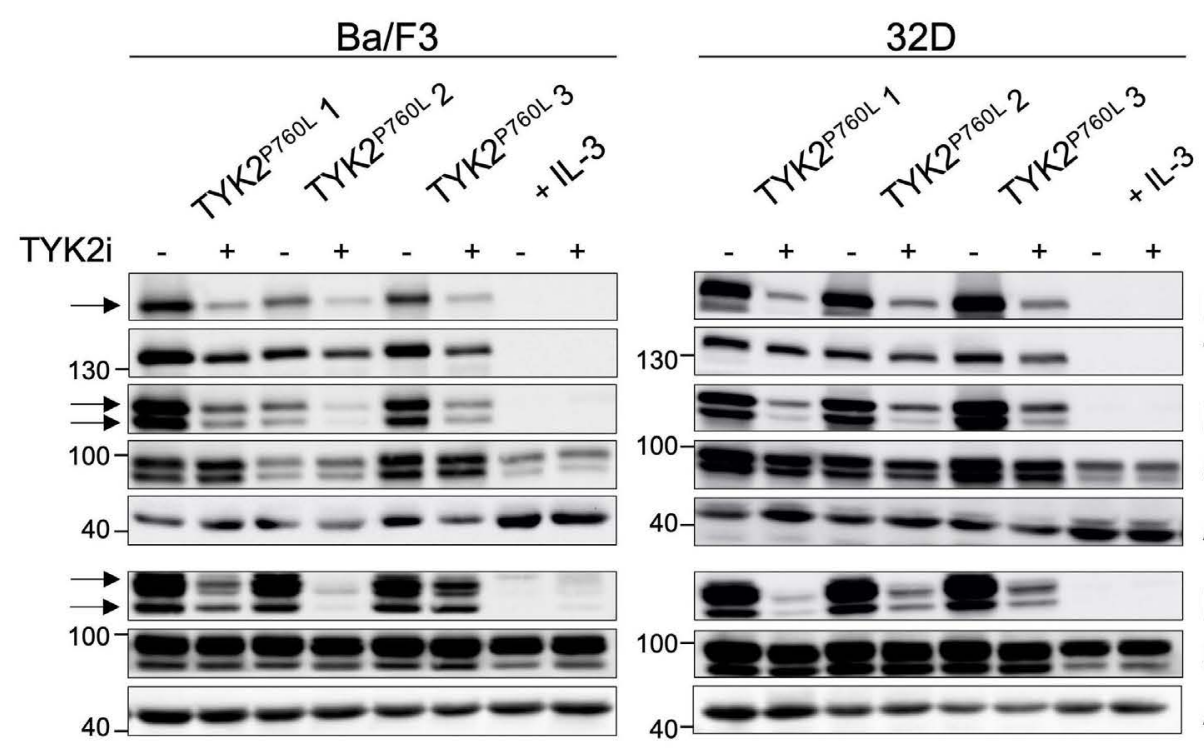

$\square$ earlya

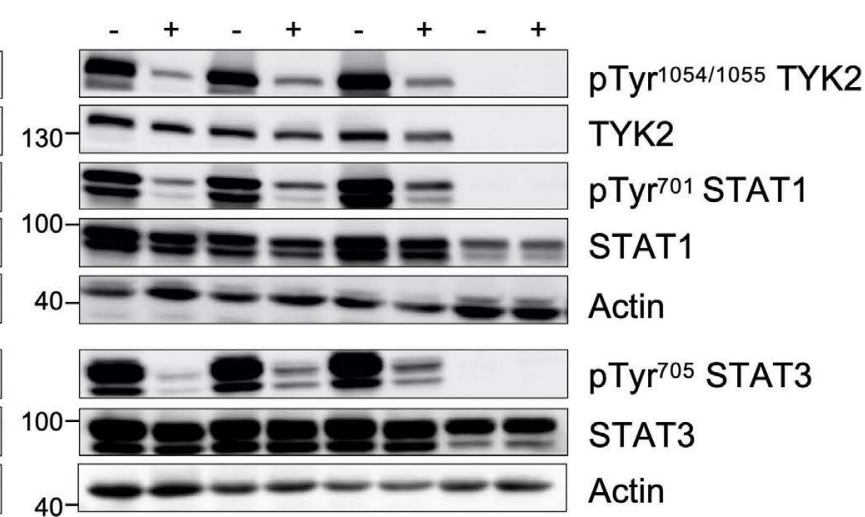

Figure 4. Viability of TYK2 P760L-transformed hematopoietic cell lines upon TYK2 inhibition and effect on signaling to STAT. (A and B) Dose response curves and half maximal inhibitory concentration (IC ${ }_{50}$ ) values of parental (black) and transformed Ba/F3 and 32D cells (shades of red) treated with deucravacitinib (TYK2inib) for 72 hours (h) supplemented with (dashed line/white filling) and without IL-3 ( $\mathrm{n} \geq 3$, in duplicates or triplicates, not all IC ${ }_{50}$ could be determined, one-way ANOVA with log transformed data). (C) Analysis of early apoptosis (Annexin $\mathrm{V}^{+}$and $7-A A D^{-}$) and late apoptosis (Annexin $\mathrm{V}^{+}$and $7-A A D^{+}$) of parental and transformed $\mathrm{Ba} / \mathrm{F} 3$ cells treated with $1 \mu \mathrm{M}$ deucravacitinib for $24 \mathrm{~h}, 48 \mathrm{~h}$ and $72 \mathrm{~h}$ ( $\mathrm{n}=3$ in duplicates). (D) Western blot of TYK2 P760L-transformed Ba/F3 and 32D cells and parental cells (with IL-3) subjected to (phospho-) TYK2-STAT1/3 analysis. Cells were treated with $1 \mu \mathrm{M}$ deucravacitinib (TYK2i) for $6 \mathrm{~h}$. Actin was used as loading control. Numbers indicate molecular weight markers in $\mathrm{kDa}$. $* * * \star P<0.0001$. 
injected with $\mathrm{Ba} / \mathrm{F} 3$ TYK2 P760L cells was 14 days and with 32D TYK2 P760L cells 32 days (Figure $3 \mathrm{H}$ ) whereas mice injected with parental $\mathrm{Ba} / \mathrm{F} 3$ or $32 \mathrm{D}$ cells did not develop leukemia. In line we found that the subcutaneous injection of $\mathrm{Ba} / \mathrm{F} 3$ or 32D TYK2 P760L cells induced local tumor growth (Figure 31 ), while no tumors or signs of disease were detected upon injection of the parental cell lines. $\mathrm{Ba} / \mathrm{F} 3$ TYK2 P760L cells engraftment was accompanied by hepatosplenomegaly caused by tumor cell infiltration (Online Supplementary Figure $S 3 \mathrm{C}$ to $H$ ) confirming the previous notion of oncogene carrying $\mathrm{Ba} / \mathrm{F} 3$ cells as migratory cells. ${ }^{27}$

Collectively, these data show that TYK2 P760L-transformed murine cells cause cancer in systemic and local transplantation setups.

\section{Pharmacological TYK2 inhibition abrogates oncogenic TYK2 P760L-driven signaling}

We next investigated how TYK2 P760L-transformed cells react to JAK inhibitors (JAKinib). Deucravacitinib (BMS986165) is a highly selective and potent allosteric TYK2inib with excellent pharmacokinetic properties across cell types and species. ${ }^{20,21,28,29}$ Drug testing was performed on $\mathrm{Ba} / \mathrm{F} 3$ and 32D TYK2 P760L cells from three outgrowth experiments (1-3; Online Supplementary Figure S3A) and on parental cells by assessing the metabolic activity. All transformed cell lines were highly sensitive to deucravacitinib with a half maximal inhibitory concentration $\left(I C_{50}\right)$ ranging from 10 to $90 \mathrm{nM}$ (Figure $4 \mathrm{~A}$ and $\mathrm{B}$ ) which is around 100 times lower than $I_{50}$ values of control cell lines. Culture of $\mathrm{Ba} / \mathrm{F} 3$ and $32 \mathrm{D}$ TYK2 P76OL cells in the presence of IL-3 reversed the effect of TYK2 inhibition, indicating that factor-independent growth was governed by mutated TYK2 (Figure 4A and B). Treatment of Ba/F3 TYK2 P760L cells with deucravacitinib induced apoptosis (Figure 4C; Online Supplementary Figure $4 A$ ) and GO cell cycle arrest (Online Supplementary Figure $4 B$ ). The efficacy of deucravacitinib was confirmed by western blotting that showed the decreased phosphorylation state of TYK2 and downstream STAT1/3 (Figure 4D).

In order to demonstrate the specificity of TYK2 inhibition in oncogenic transformed $\mathrm{Ba} / \mathrm{F} 3$ cells and to exclude potential off-target effects of deucravacitinib, we tested three additional $\mathrm{Ba} / \mathrm{F} 3$ cell lines driven by known oncogenes, i.e., TEL-JAK2, STAT5B N642H and BCR-ABL1 p210. In these settings $I C_{50}$ values were comparable to parental $\mathrm{Ba} / \mathrm{F} 3$ cells cultured with IL-3 (Online Supplementary Figure $S 5 A$ ). These experiments prove that deucravacitinib is selective for TYK2 and specifically interferes with TYK2dependent transformation. Further support for the unique function of TYK2 stems from experiments with the JAK1/3 inhibitor tofacitinib, ${ }^{30}$ the JAK1/2 inhibitor ruxolitinib ${ }^{31}$ and the JAK1 inhibitor filgotinib. ${ }^{32}$ None of these JAKinib when employed at specific concentrations - significantly decreased the metabolic activity of TYK2 P760L-transformed cells (except Ba/F3 TYK2 P760L 1 with ruxolitinib) compared to parental $\mathrm{Ba} / \mathrm{F} 3$ and $32 \mathrm{D}$ cells (Online Supplementary Figure $S 5 B$ to $G$ ).

In summary we confirmed that only the TYK2-selective drug deucravacitinib efficiently reduces the cell viability of TYK2 P760L-transformed cells. This underscores the key role of TYK2 in maintaining the transformed state and excludes a major contribution of other JAK family members.

\section{TYK2 P760L signaling co-operates with the PI3K/AKT/mTOR and CDK4/6 pathways}

In order to understand whether and how oncogenic TYK2 P760L co-operates with other signaling pathways we designed a drug screen with commercial libraries of 680 approved or investigational kinase inhibitors (Figure 5A). A first screen in Ba/F3 TYK2 P760L cells identified 246 drugs that reduce metabolically active cells to less than $30 \%$ compared to the control. In a second round, we included the $\mathrm{Ba} / \mathrm{F} 3$ parental cell line as control and counter screen and looked for drugs that specifically block the TYK2 P760L-expressing cells. This narrowed the output down to 34 drugs, of which several target the $\mathrm{PI} 3 \mathrm{~K} / \mathrm{AKT} / \mathrm{mTOR}$ (8/34 drugs) and CDK4/6 (3/34 drugs) pathways (Figure 5A; Online Supplementary Table S4). Involvement of these pathways is also supported by increased phosphorylation of AKT in both TYK2 P760L-transformed cell lines and increased levels of CDK6 in transformed 32D cells. No changes in the mTOR target 4EBP1 were observed (Figure 5B).

The compound olverembatinib (GZD824) scored as top hit of the screen (Online Supplementary Table S4). Olverembatinib was initially described as inhibitor of the BCR-ABL1 fusion kinase but also interferes with PI3K/AKT and SRC kinase signaling. ${ }^{33}$ In order to validate our screening results, we determined dose response curves with inhibitors for the PI3K/AKT/mTOR or CDK4/6 pathway and used everolimus, vistusertib, olverembatinib, LY294002, abemaciclib and palbociclib (Figure 5A). We included the TYK2 P760Ltransformed $\mathrm{Ba} / \mathrm{F} 3$ and $32 \mathrm{D}$ cells, and BCR-ABL1-, TELJAK2- and STAT5B N642H-transformed Ba/F3 cells as controls. TYK2 P760L-transformed $\mathrm{Ba} / \mathrm{F} 3$ and $32 \mathrm{D}$ cells were more sensitive to all tested drugs compared to controls (Figure $5 \mathrm{C}$ to E; Online Supplementary Figures S6A to $C$ and STA to $L$ ). MTOR inhibitors everolimus and vistusertib were the most efficacious drugs with highest difference between transformed and parental $\mathrm{Ba} / \mathrm{F} 3$ cell lines (Figure 5C and Online Supplementary Figure S6A). At the effective concentrations determined in the dose-response curves all tested drugs prevented IL-3-independent growth (Online Supplementary Figure S $8 A$ and $B$ ).

Cancer therapy regimens rely on drug combinatorial treatments. ${ }^{22}$ We thus combined the TYK2 inhibitor (deucravacitinib) with all tested co-operating inhibitors and 
A

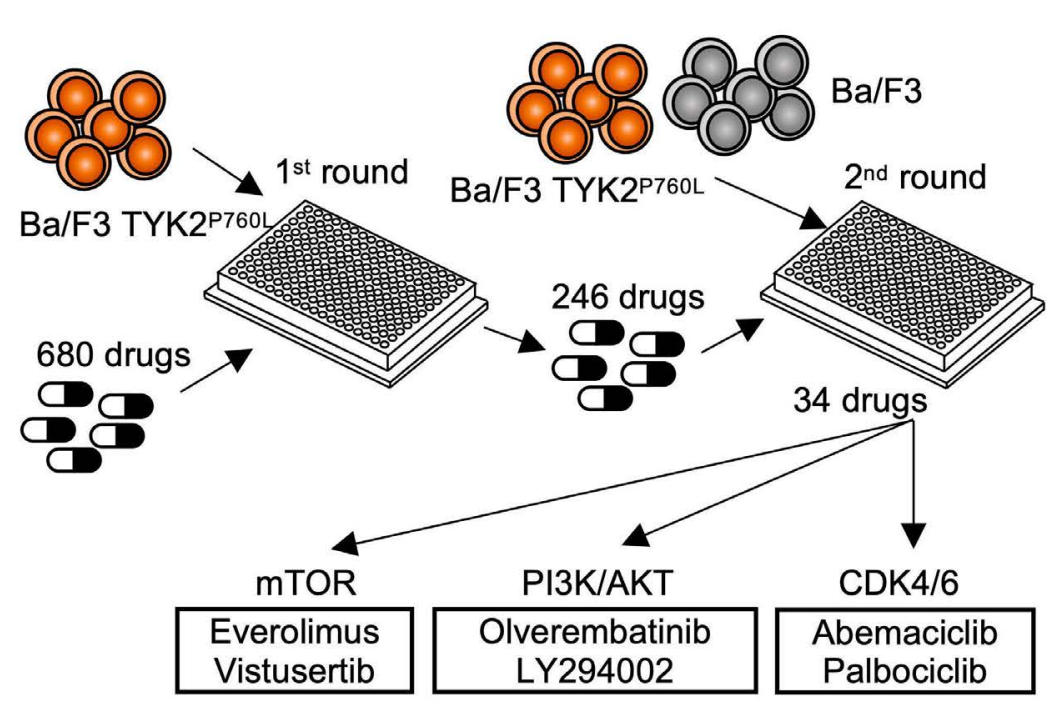

B

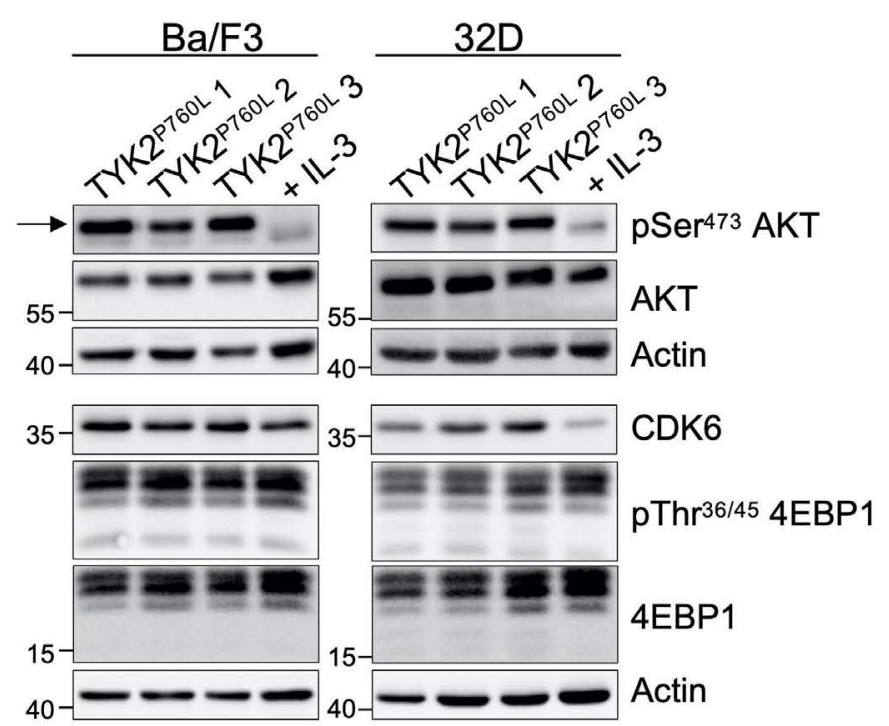

C

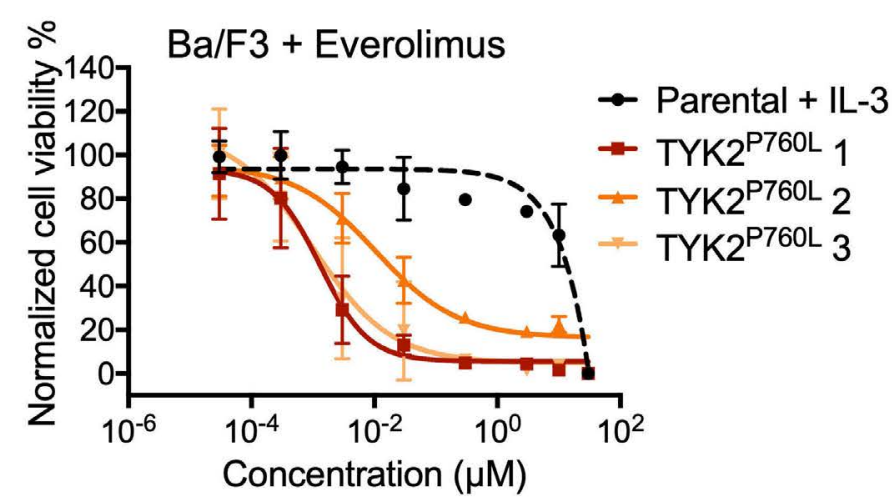

D

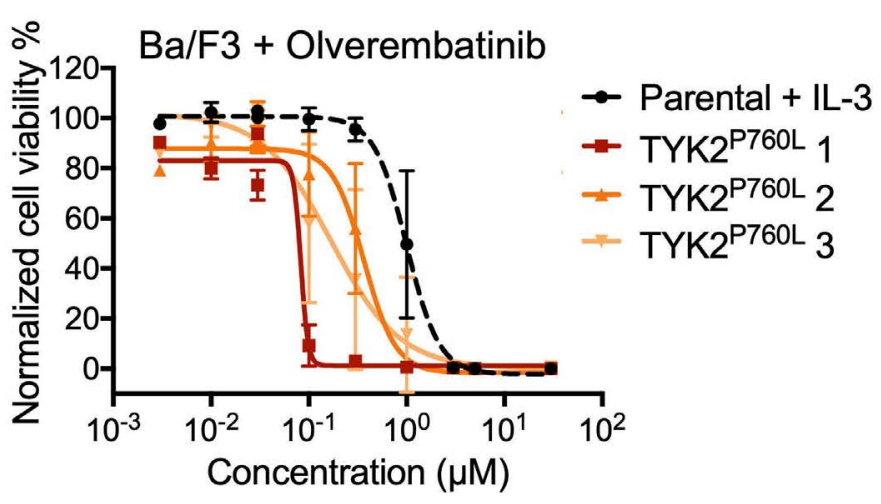

E

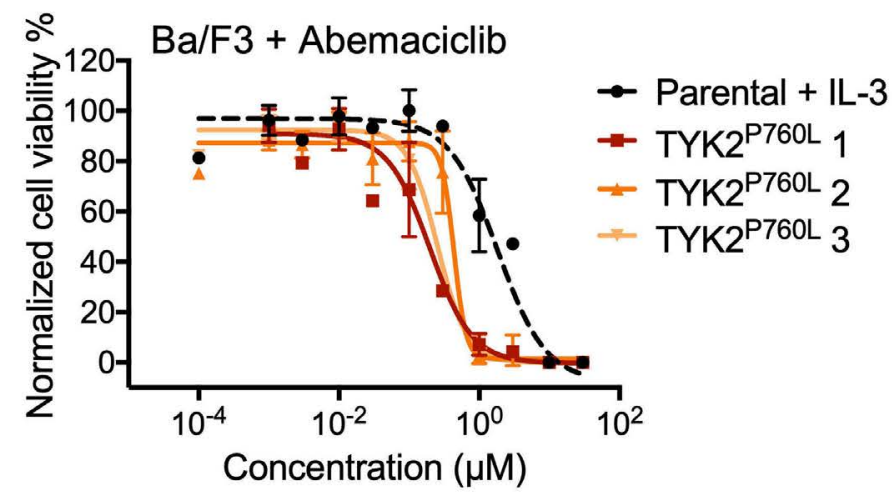

$\mathbf{F}$
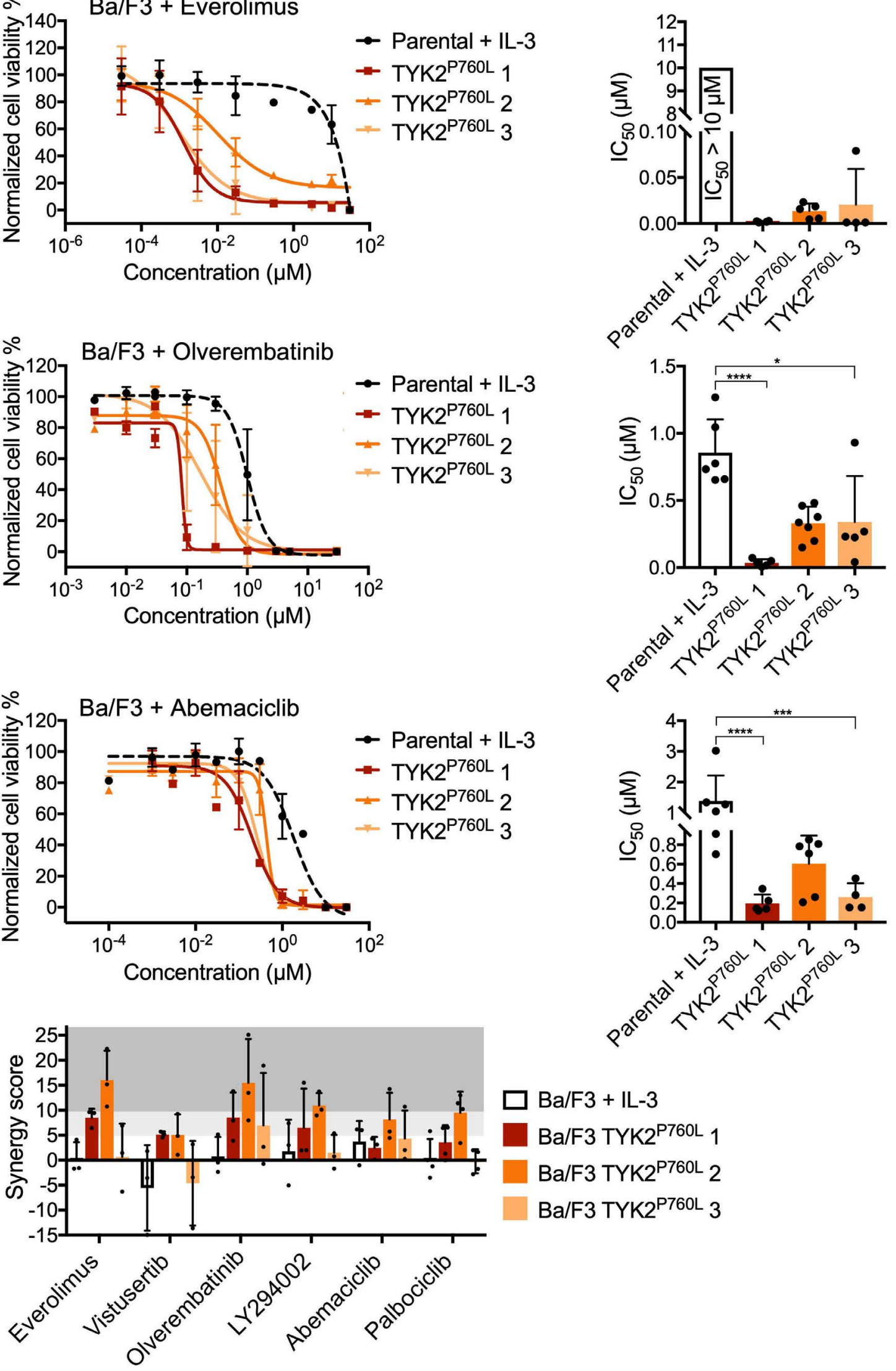

Continued on following page. 
Figure 5. Drug screen of TYK2 P760L-expressing cells and cross-talk with other kinase-dependent pathways. (A) Schematic outline of drug screen: Ba/F3 TYK2 P760L cells of the first outgrowth experiment were treated with 680 kinase inhibitors in a high throughput screen. In the second round 246 drugs identified ( $<30 \%$ viability of control) were used for comparison of Ba/F3 TYK2 P760L and parental cells. Among the top hits were mainly drugs targeting the mTOR, PI3K/AKT and CDK4/6 pathway. These targets were validated with the indicated drugs. (B) Western blot of TYK2 P760L-transformed Ba/F3 and 32D cells and parental cells (with IL-3) subjected to (phospho-) AKT, 4EBP1 and CDK6 analysis. Actin was used as loading control. Numbers indicate molecular weight markers in $\mathrm{kDa}$. ( $\mathrm{C}$ to $\mathrm{E}$ ) Validation of screen result with dose-response curves and half maximal inhibitory concentration $\left(\mathrm{IC}_{50}\right.$ ) values of parental (black dashed line/white filling, with IL-3) and transformed Ba/F3 cells (shades of red) treated for 72 hours ( $\mathrm{n} \geq 3$, in duplicates, not all IC ${ }_{50}$ could be determined, one-way ANOVA with log transformed data). (F) Synergy scores ( $n \geq 3$, in duplicates) calculated with the zero interaction potency (ZIP) model. Light grey shows additivity, dark grey synergy. $* P<0.05, * \star * P<0.001, * \star * * P<0.0001$.

calculated the synergy score by the zero interaction potency (ZIP) method. ${ }^{34,35}$ For Ba/F3 TYK2 P760L cells 11/18 and for 32D TYK2 P760L cells 16/18 combinations reached a synergy score higher than five indicating additive effects. Synergy scores $\geq 10$ were found in Ba/F3 TYK2 P760L for the combination with PI3K/AKT/mTOR inhibitors (everolimus, LY294002 and olverembatinib), in 32D TYK2 P760L cells for the combination with the PI3K/AKT inhibitor LY294002 and the CDK4/6 inhibitor abemaciclib (Figure 5F; Online Supplementary Figure S8C to $\mathrm{H}$ ).

Taken together, oncogenic TYK2 and co-operating pathways were successfully blocked by combinatorial treatment in murine cellular models.

\section{Combined treatment with TYK2 and mTOR or CDK4/6 inhibitors decreases viability of TYK2 P760L xenograft cells}

In order to translate these results in a patient-related setting, we analyzed the combinatorial treatments with deucravacitinib in human PDX cells harboring TYK2 P76OL (Online Supplementary Figure S9A) co-cultured with human hTERT mesenchymal stem cells. The patient carrying the TYK2 P760L mutation first developed a leukemia classified as a chromosomal rearranged pre-B-ALL (leukemia 1) and subsequently a hyperdiploid common B-ALL (leukemia 2). ${ }^{17}$ PDX cells were generated from both leukemias and were co-treated with increasing concentrations of deucravacitinib and inhibitors of the identified co-operating pathways. Adding deucravacitinib to mTOR and CDK4/6 inhibitors resulted in a left-shift of the dose response curves (except for palbociclib in PDX cells leukemia 1) (Figure 6A to D; Online Supplementary Figure $S 9 B$ to $E$ ). For both leukemias the synergy score was higher than 5 for the combination with abemaciclib (Figure 6C and D) and for the leukemia 2 the combination with vistusertib and palbociclib (Online Supplementary Figure S9C and $E$ ). The most synergistic area scores, which indicate the peak of the synergy matrix, were $\geq 5$ for both leukemias with all treatments except leukemia 1 with palbociclib and $\geq 10$ for both leukemias with abemaciclib, leukemia 1 with everolimus and leukemia 2 with vistusertib (Figure 6A to $\mathrm{D}$; Online Supplementary Figure $S 9 B$ to $E$ ). Combinations with PI3K/AKT inhibitors (olverembatinib and LY294002) failed to display synergism (data not shown).
These data show that the TYK2 inhibitor improves the efficacy of mTOR and CDK4/6 inhibitors in patient cells.

\section{Discussion}

We investigated the TYK2 P760L germline GOF mutation that was found in childhood $\mathrm{B}-\mathrm{ALL}^{17}$ with regard to its oncogenic and druggable properties. Using in vitro and in vivo models we demonstrate that constitutively active human TYK2 P760L conferred growth/proliferation advantages on primary and immortalized hematopoietic cells. TYK2 P760L-harboring murine cells led to leukemic disease upon transplantation. A selective TYK2inib, but not JAKinib targeting JAK1-3, was highly efficacious in decreasing the metabolic activity of TYK2 P760L-transformed cells. A kinase inhibitor screen identified pathways co-operating with oncogenic TYK2 and established combinatorial TYK2 inhibition with mTOR or CDK4/6 pathway blockade as therapeutic option to eradicate TYK2-driven leukemia.

We established the oncogenic potential of the TYK2 P760L GOF mutation in different cell systems ranging from primary BM cells to hematopoietic cell lines of various differentiation stages as exemplified by enhanced colony formation or growth factor-independent proliferation in vitro and migratory properties in vivo. Thus, the germline TYK2 mutation qualifies for inclusion in the expanding list of cancer driver gene mutations. ${ }^{36}$ This mutation is predicted to change the structure of the conserved DPG motif of JAK pseudokinases and thereby attenuates the inhibitory function. ${ }^{17}$ The DPG motif replaces the DFG motif of kinases and is involved in several non-covalent interactions within the $\mathrm{JH} 2$ domain. ${ }^{37}$ DPG mutations of other JAK listed in cancer databases are not further characterized. An activating JAK3 V674A mutation (adjacent to the DPG motif) found in T-ALL patients has been shown to cause leukemia in mice. ${ }^{38}$ Under the conditions analyzed, constitutively active TYK2 did not suffice to immortalize primary cells or allow for factor-independent growth of all cell lines tested. This is in line with the properties of germline-transmitted leukemia-predisposing mutations that frequently block the differentiation of lymphoid cells but need co-operating factors that enhance indefinite survival for malignant transformation. ${ }^{39}$ It is also in line with the reported weaker malignant 
transformation capacity of a given activating mutation of TYK2 compared to other JAK. ${ }^{40,41}$

As previously shown TYK2 P760L expression leads to constitutive STAT1/3 signaling which is described to shape the oncogenic transcriptome. ${ }^{8,17}$ We provide strong evidence that these signals stem directly from TYK2 activity as introduction of a kinase-inactivating mutation reverts STAT activation and cellular transformation. In addition, our studies with JAKinib revealed that only the TYK2-specific inhibitor deucravacitinib and not inhibitors with higher sel-
A

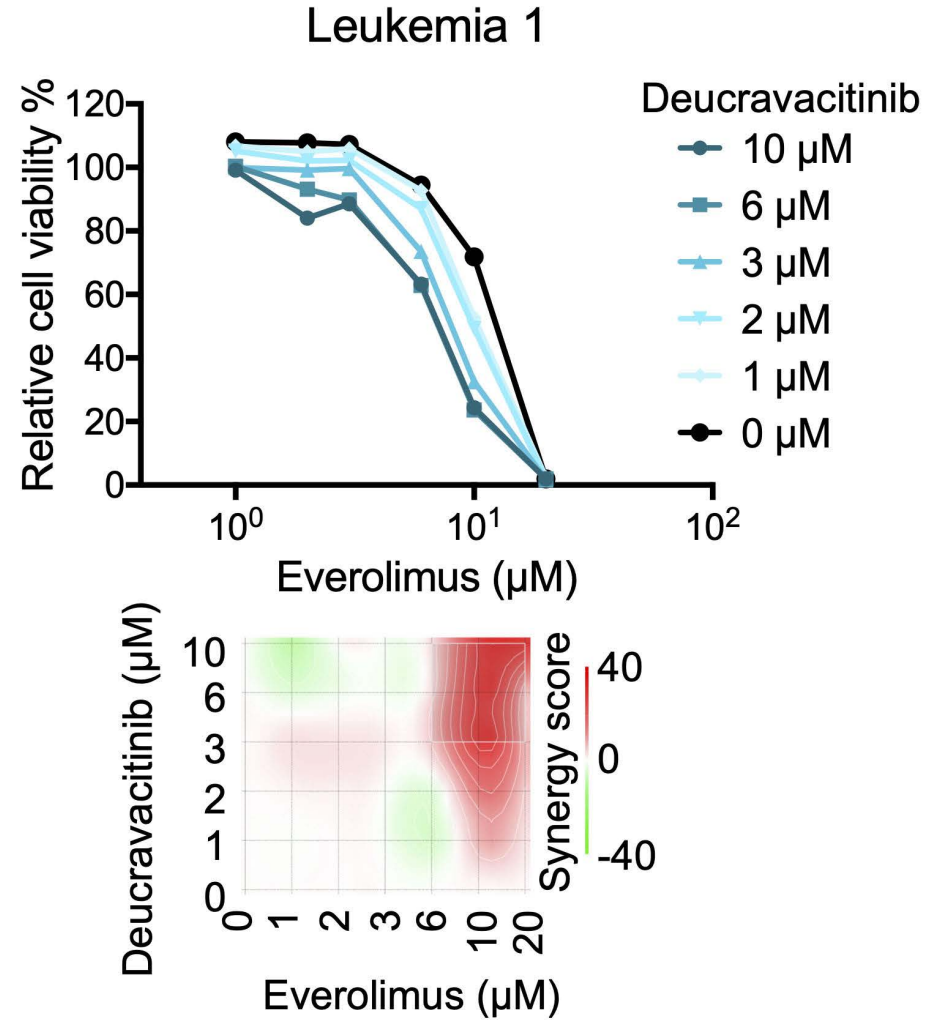

Synergy score: 4.01

Most synergistic area score: 15.77
B

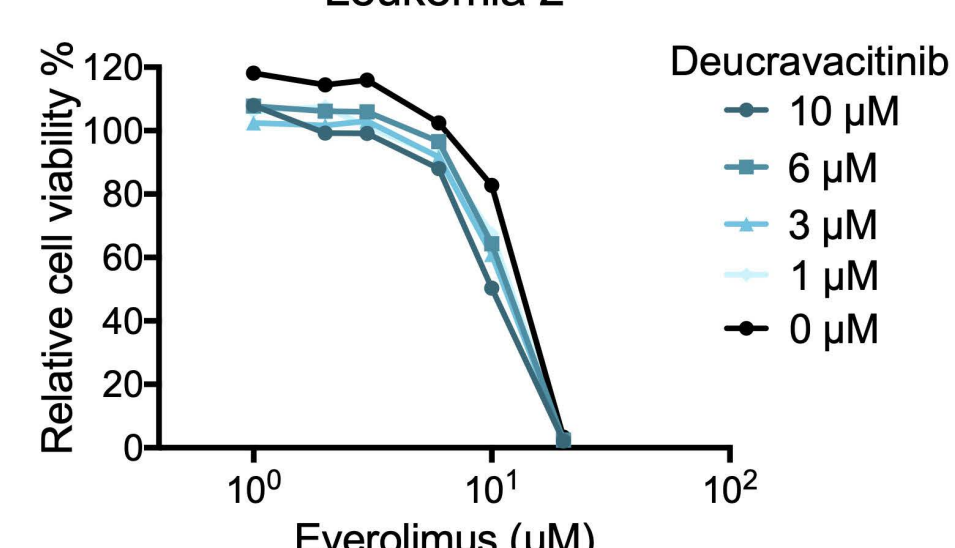

Synergy score: $\mathbf{4 . 7 8}$

Most synergistic area score: $\mathbf{7 . 7 2}$

C Leukemia 1

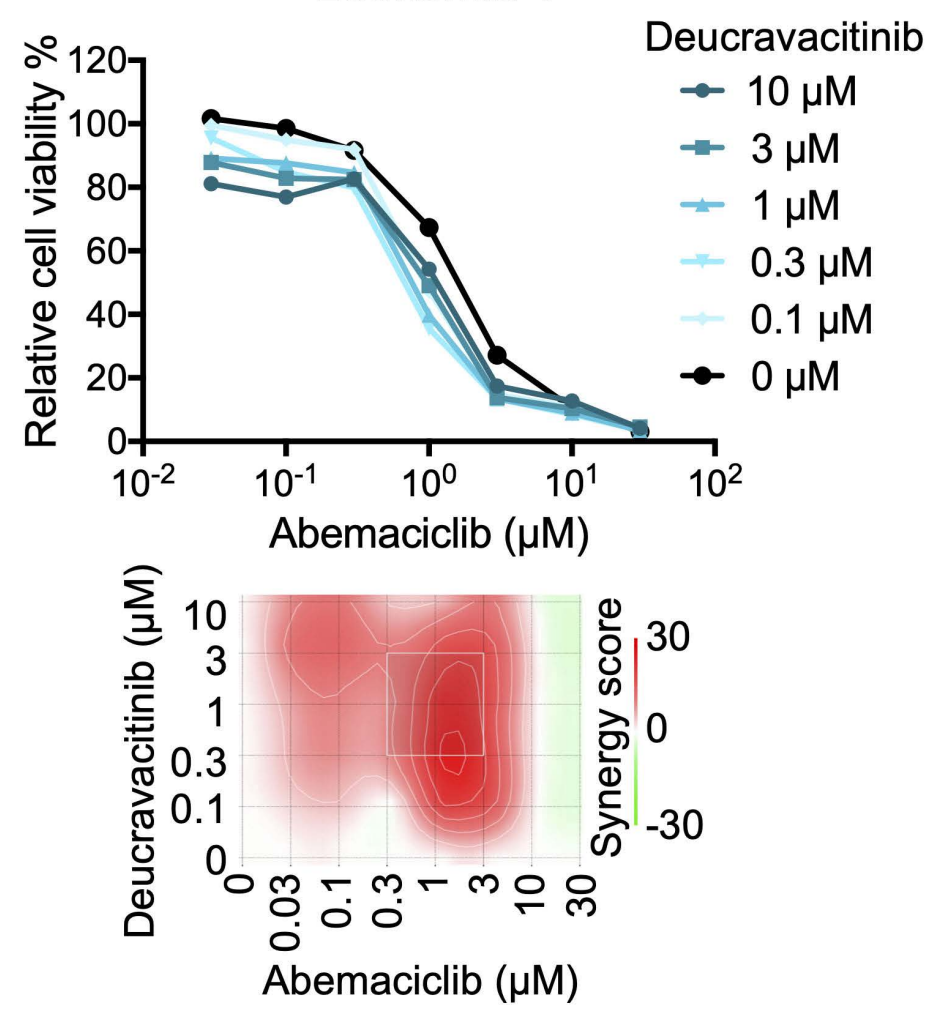

Synergy score: $\mathbf{7 . 9 2}$

Most synergistic area score: $\mathbf{1 6 . 4 2}$
D
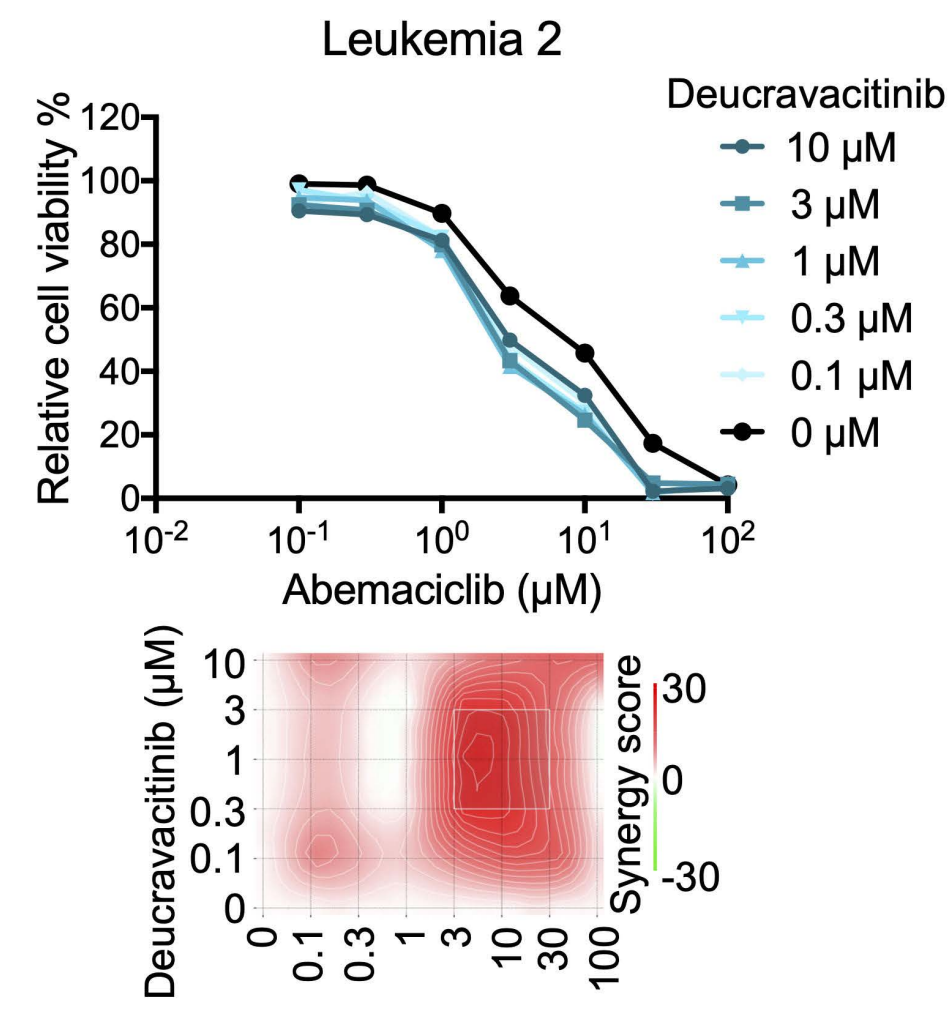

Abemaciclib $(\mu \mathrm{M})$

\section{Synergy score: $\mathbf{8 . 6 1}$}

Most synergistic area score: $\mathbf{1 8 . 7 9}$

Figure 6. TYK2 P760L patient-derived xenograft cells under combinatorial drug treatment. (A to D) Cell viability curves of hTERT mesenchymal stem cells co-cultured patient-derived xenograft cells of leukemia 1 and leukemia 2 treated with different concentrations of the indicated drug and different concentrations of deucravacitinib (shades of blue) for 72 hours. A synergy map is shown for each drug combination. Synergy scores and most synergistic area score were calculated with the zero interaction potency (ZIP) model $(n=1)$. Red shows synergism and green antagonism. 
ectivity for JAK1-3 showed efficacy in cell viability assays with TYK2 P760L-transformed cells.

Deucravacitinib belongs to a novel class of JAKinib as it does not block the enzymatic activity in the $\mathrm{JH} 1$ domain but rather stabilizes the regulatory pseudokinase domain $\mathrm{JH} 2 .^{19-21}$ The inhibitor was shown to bind also the $\mathrm{JH} 2$ domain of JAK1, albeit with less affinity and selectivity. ${ }^{20,21}$ Importantly, the biochemical data translate in a weak activity against JAK1-dependent signaling. ${ }^{20,21,28,29}$ The efficiency of JH1-targeting TYK2 inhibitors in leukemia treatment has been shown for T-ALL xenografts. ${ }^{42}$ We are the first to prove the successful application of a JH2-targeting drug on a hyperactive TYK2 carrying the mutation in the JH2 domain. This might be important for future development of JAKinib.

Kinases represent one of the largest groups of druggable targets as they drive key signaling pathways and aberrant kinase activity leads or contributes to cancer onset and progression. ${ }^{43}$ In order to identify co-operation of oncogenic TYK2 with other kinase-driven pathways, we have screened inhibitor libraries using TYK2 P760L-transformed cells and identified the most potent hits in the $\mathrm{PI}$ IKK/AKT/mTOR and CDK4/6 signaling axes. TYK2-dependent activation of PI3K and a crosstalk between JAK/STAT and mTOR signaling is well established. ${ }^{3,44,45}$ Aberrant TYK2-PI3K activity has been also reported for prostate cancer $^{46}$ and T-ALL. ${ }^{11}$ The PI3K/AKT/mTOR pathway is frequently activated in ALL and specific inhibitors alone or in combination with JAKinib have been successfully applied in Ph-like ALL. ${ }^{47}$ At the molecular level CDK4/6 is reported to connect cell cycle progression with cell growth via mTOR activation, which may explain the efficacy of combined blocking of CDK4/6 and of PI3K/AKT/mTOR in solid tumors. ${ }^{48} \mathrm{~A}$ direct connection between CDK4/6 and TYK2 has not been described. However, STAT3, downstream of TYK2 P760L, is known to activate CDK4/6 during the cell cycle progression. ${ }^{49}$ CDK6 was shown to interact with STAT3 in tumorigenesis ${ }^{50}$ and CDK6 hyperactivation has been reported in ALL. ${ }^{51}$ Importantly, our combinatorial treatment of the TYK2 inhibitor with mTOR or CDK4/6 inhibitors was successfully applied in the TYK2 GOF PDX cells. Ex vivo assays tend to underestimate the effects of cell cycle drugs as even stromal co-cultures do not fully support proliferation of ALL xenografts..$^{52}$ In this context it is important to note that CDK6 also exhibits kinase- and cell cycle-independent functions in tumorigenesis. ${ }^{53,54}$ In summary, we provide proof of TYK2 acting as an oncogene in hematologic malignancies by firmly establishing the oncogenic potential of TYK2 germline mutations, revealing that TYK2 P760L is involved in leukemia progression. Moreover, our results indicate that combinatorial kinase inhibition in acute leukemia could be a valid strategy to combat hyperactivated TYK2-mutated leukemia.

\section{Disclosures}

No conficts of interest to disclose.

\section{Contributions}

$K W$ performed and analyzed most experiments; $S M-M, B S$ and $M M$ supervised the project; $S M-M, M B, K M, B M, T B$ and $A O$ provided technical support; $A K$ and SK performed and analyzed the kinase inhibitor screen; SM-M, DSVIS, CL and $A P$ helped with in vivo mouse studies; CV helped with statistical analysis; DV performed some experiments; RM and $V S$ provided crucial material and reagents; $S M-M, R M, B S$, $V S, F N V L, R P K$ and MM were involved in study design and provided crucial scientific input; KW and MM wrote the manuscript with input from all authors. All authors approved the manuscript.

\section{Acknowledgments}

The authors thank Marion Bokor for histological sample preparations and H\&E staining, Philipp Jodl for technical support, the mouse facility personnel for their help with in vivo experiments, Thomas Kolbe for provision of some NSG mice and Michael Dworzak for provision of a cell line.

\section{Funding}

This work was supported by the Austrian Science Fund (FWF) funded DK W1212 PhD program "Inflammation and Immunity" and the Special Research Program SFB F6101 and F6106 (BS and MM), F6105 (RM) and F6107 (VS).

\section{Data-sharing statement}

All novel reagents mentioned are available upon justified request.

\section{References}

1. Vogelstein B, Papadopoulos N, Velculescu VE, et al. Cancer genome landscapes. Science. 2013;339(6127):1546-1558.

2. Sanchez-Vega F, Mina M, Armenia J, et al. Oncogenic signaling pathways in the cancer genome atlas. Cell. 2018;173(2):321-337.

3. Saleiro D, Platanias LC. Intersection of mTOR and STAT signaling in immunity. Trends Immunol. 2015;36(1):21-29.

4. Steelman LS, Pohnert SC, Shelton JG, et al. JAK/STAT, Raf/MEK/ERK, PI3K/Akt and BCR-ABL in cell cycle progression and leukemogenesis. Leukemia. 2004;18(2):189-218.

5. O'Shea JJ, Schwartz DM, Villarino AV, et al. The JAK-STAT pathway: impact on human disease and therapeutic intervention. Annu Rev Med. 2015;66:311-328.

6. Hammaren HM, Virtanen AT, Raivola J, et al. The regulation of JAKs in cytokine signaling and its breakdown in disease.

Cytokine. 2019;118:48-63.

7. Vainchenker W, Constantinescu SN. JAK/STAT signaling in 
hematological malignancies. Oncogene. 2013;32(21):2601-2613.

8. Wöss K, Simonovic N, Strobl B, et al. TYK2: an upstream kinase of STATs in cancer. Cancers (Basel). 2019;11(11):1728.

9. Pellenz FM, Dieter C, Lemos NE, et al. Association of TYK2 polymorphisms with autoimmune diseases: A comprehensive and updated systematic review with meta-analysis. Genet Mol Biol. 2021;44(2):e20200425.

10. Strobl B, Stoiber D, Sexl V, et al. Tyrosine kinase 2 (TYK2) in cytokine signalling and host immunity. Front Biosci (Landmark Ed). 2011;16:3214-3232.

11. Sanda T, Tyner JW, Gutierrez A, et al. TYK2-STAT1-BCL2 pathway dependence in T-cell acute lymphoblastic leukemia. Cancer Discov. 2013;3(5):564-577.

12. Velusamy T, Kiel MJ, Sahasrabuddhe AA, et al. A novel recurrent NPM1-TYK2 gene fusion in cutaneous CD30-positive lymphoproliferative disorders. Blood. 2014;124(25):3768-3771.

13. Crescenzo R, Abate F, Lasorsa E, et al. Convergent mutations and kinase fusions lead to oncogenic STAT3 activation in anaplastic large cell lymphoma. Cancer Cell. 2015;27(4):516-532.

14. Roberts KG, Li Y, Payne-Turner D, et al. Targetable kinaseactivating lesions in Ph-like acute lymphoblastic leukemia. $\mathrm{N}$ Engl J Med. 2014;371(11):1005-1015.

15. Prutsch N, Gurnhofer E, Suske T, et al. Dependency on the TYK2/STAT1/MCL1 axis in anaplastic large cell lymphoma. Leukemia. 2019;33(3):696-709.

16. Pui $\mathrm{CH}$, Nichols KE, Yang JJ. Somatic and germline genomics in paediatric acute lymphoblastic leukaemia. Nat Rev Clin Oncol. 2019;16(4):227-240.

17. Waanders E, Scheijen B, Jongmans MC, et al. Germline activating TYK2 mutations in pediatric patients with two primary acute lymphoblastic leukemia occurrences. Leukemia. 2017;31(4):821-828.

18. Bryan MC, Rajapaksa NS. Kinase inhibitors for the treatment of immunological disorders: recent advances. J Med Chem. 2018;61(20):9030-9058.

19. Tokarski JS, Zupa-Fernandez A, Tredup JA, et al. Tyrosine kinase 2-mediated signal transduction in T lymphocytes is blocked by pharmacological stabilization of its pseudokinase domain. J Biol Chem. 2015;290(17):11061-11074.

20. Burke JR, Cheng L, Gillooly KM, et al. Autoimmune pathways in mice and humans are blocked by pharmacological stabilization of the TYK2 pseudokinase domain. Sci Transl Med. 2019;11(502):eaaw1736

21. Wrobleski ST, Moslin R, Lin S, et al. Highly selective inhibition of tyrosine kinase 2 (TYK2) for the treatment of autoimmune diseases: discovery of the allosteric inhibitor BMS-986165. J Med Chem. 2019;62(20):8973-8995.

22. Al-Lazikani B, Banerji U, Workman P. Combinatorial drug therapy for cancer in the post-genomic era. Nat Biotechnol. 2012;30(7):679-692.

23. Klein $\mathrm{K}$, Stoiber $\mathrm{D}$, Sexl V, et al. Untwining anti-tumor and immunosuppressive effects of JAK inhibitors-A strategy for hematological malignancies? Cancers (Basel). 2021;13(11):2611.

24. Shultz LD, Lyons BL, Burzenski LM, et al. Human lymphoid and myeloid cell development in NOD/LtSz-scid IL2R gamma null mice engrafted with mobilized human hemopoietic stem cells. J Immunol. 2005;174(10):6477-6489.

25. Cheng $H$, Zheng Z, Cheng T. New paradigms on hematopoietic stem cell differentiation. Protein Cell. 2020;11(1):34-44.

26. Linnekin D, Mou S, Deberry CS, et al. Stem cell factor, the JAKSTAT pathway and signal transduction. Leuk Lymphoma. 1997;27(5-6):439-444.

27. Funakoshi-Tago M, Tago K, Sumi $\mathrm{K}$, et al. The acute lymphoblastic leukemia-associated JAK2 L611S mutant induces tumorigenesis in nude mice. $\mathrm{J}$ Biol Chem. 2009;284(19):12680-12690.

28. Chimalakonda A, Burke J, Cheng L, et al. Selectivity profile of the tyrosine kinase 2 inhibitor deucravacitinib compared with janus kinase 1/2/3 inhibitors. Dermatol Ther (Heidelb). 2021;11(5):1763-1776.

29. Catlett IM, Hu Y, Gao L, et al. Molecular and clinical effects of selective TYK2 inhibition with deucravacitinib in psoriasis. $J$ Allergy Clin Immunol. 2021;S0091-6749(21)01690-0.

30. Flanagan ME, Blumenkopf TA, Brissette WH, et al. Discovery of CP-690,550: a potent and selective Janus kinase (JAK) inhibitor for the treatment of autoimmune diseases and organ transplant rejection. J Med Chem. 2010;53(24):8468-8484.

31. Quintas-Cardama A, Vaddi K, Liu P, et al. Preclinical characterization of the selective JAK1/2 inhibitor INCB018424: therapeutic implications for the treatment of myeloproliferative neoplasms. Blood. 2010;115(15):3109-3117.

32. Van Rompaey L, Galien R, van der Aar EM, et al. Preclinical characterization of GLPG0634, a selective inhibitor of JAK1, for the treatment of inflammatory diseases. J Immunol. 2013;191(7):3568-3577.

33. Ye W, Jiang Z, Lu X, et al. GZD824 suppresses the growth of human $B$ cell precursor acute lymphoblastic leukemia cells by inhibiting the SRC kinase and PI3K/AKT pathways. Oncotarget. 2017;8(50):87002-87015.

34. Yadav B, Wennerberg K, Aittokallio T, et al. Searching for drug synergy in complex dose-response landscapes using an interaction potency model. Comput Struct Biotechnol J. 2015;13:504-513.

35. lanevski A, Giri AK, Aittokallio T. SynergyFinder 2.0: visual analytics of multi-drug combination synergies. Nucleic Acids Res. 2020;48(W1):W488-W493.

36. Martinez-Jimenez F, Muinos F, Sentis I, et al. A compendium of mutational cancer driver genes. Nat Rev Cancer. 2020;20(10):555-572.

37. Min X, Ungureanu D, Maxwell S, et al. Structural and functional characterization of the $\mathrm{JH} 2$ pseudokinase domain of JAK family tyrosine kinase 2 (TYK2). J Biol Chem. 2015;290(45):27261-27270.

38. Degryse $S$, de Bock CE, Cox L, et al. JAK3 mutants transform hematopoietic cells through JAK1 activation, causing T-cell acute lymphoblastic leukemia in a mouse model. Blood. 2014;124(20):3092-3100.

39. Tijchon E, Havinga J, van Leeuwen FN, Scheijen B. B-lineage transcription factors and cooperating gene lesions required for leukemia development. Leukemia. 2013;27(3):541-552.

40. Staerk J, Kallin A, Demoulin JB, et al. JAK1 and Tyk2 activation by the homologous polycythemia vera JAK2 V617F mutation: cross-talk with IGF1 receptor. J Biol Chem. 2005;280(51):41893-41899.

41. Shide K, Shimoda K, Kamezaki K, et al. Tyk2 mutation homologous to V617F Jak2 is not found in essential thrombocythaemia, although it induces constitutive signaling and growth factor independence. Leuk Res. 2007;31(8):1077-1084.

42. Akahane K, Li Z, Etchin J, et al. Anti-leukaemic activity of the TYK2 selective inhibitor NDI-031301 in T-cell acute lymphoblastic leukaemia. Br J Haematol. 2017;177(2):271-282.

43. Klaeger S, Heinzlmeir S, Wilhelm M, et al. The target landscape of clinical kinase drugs. Science. 2017;358(6367):eaan4368.

44. Rani MR, Leaman DW, Han Y, et al. Catalytically active TYK2 is essential for interferon-beta-mediated phosphorylation of STAT3 and interferon-alpha receptor-1 (IFNAR-1) but not for activation of phosphoinositol 3-kinase. J Biol Chem. 1999;274(45):32507-32511. 
45. Kusch A, Tkachuk S, Haller $\mathrm{H}$, et al. Urokinase stimulates human vascular smooth muscle cell migration via a phosphatidylinositol 3-kinase-Tyk2 interaction. J Biol Chem. 2000;275(50):39466-39473.

46. Ide H, Nakagawa T, Terado Y, et al. Tyk2 expression and its signaling enhances the invasiveness of prostate cancer cells. Biochem Biophys Res Commun. 2008;369(2):292-296.

47. Tasian SK, Teachey DT, Li Y, et al. Potent efficacy of combined $\mathrm{PI} 3 \mathrm{~K} / \mathrm{mTOR}$ and JAK or ABL inhibition in murine xenograft models of Ph-like acute lymphoblastic leukemia. Blood. 2017;129(2):177-187.

48. Romero-Pozuelo J, Figlia G, Kaya O, et al. Cdk4 and Cdk6 couple the cell-cycle machinery to cell growth via mTORC1. Cell Rep. 2020;31(2):107504.

49. Fukada T, Ohtani T, Yoshida Y, et al. STAT3 orchestrates contradictory signals in cytokine-induced G1 to $S$ cell-cycle transition. EMBO J. 1998;17(22):6670-6677.

50. Kollmann K, Heller G, Schneckenleithner C, et al. A kinaseindependent function of CDK6 links the cell cycle to tumor angiogenesis. Cancer Cell. 2013;24(2):167-181.

51. Nebenfuehr S, Kollmann K, Sexl V. The role of CDK6 in cancer. Int J Cancer. 2020;147(11):2988-2995.

52. Frismantas $V$, Dobay MP, Rinaldi A, et al. Ex vivo drug response profiling detects recurrent sensitivity patterns in drug-resistant acute lymphoblastic leukemia. Blood. 2017;129(11):e26-e37.

53. Bellutti F, Tigan AS, Nebenfuehr S, et al. CDK6 antagonizes p53-induced responses during tumorigenesis. Cancer Discov. 2018;8(7):884-897.

54. Uras IZ, Maurer B, Nivarthi H, et al. CDK6 coordinates JAK2 (V617F) mutant MPN via NF-kappaB and apoptotic networks. Blood. 2019;133(15):1677-1690. 